Research Article

\title{
Reexamining Spatiotemporal Disparities of Financial Development in China Based on Functional Data Analysis
}

\author{
Deqing Wang $(\mathbb{D}$, Tianzhi Ye, Sihua Tian, and Xu Wang \\ Jiangsu Key Laboratory of Coal-based Greenhouse Gas Control and Utilization, School of Economics and Management, \\ China University of Mining and Technology, Xuzhou 221116, China
}

Correspondence should be addressed to Deqing Wang; dekinywang@cumt.edu.cn

Received 12 January 2021; Revised 4 March 2021; Accepted 20 March 2021; Published 17 April 2021

Academic Editor: Petr Hájek

Copyright ( 2021 Deqing Wang et al. This is an open access article distributed under the Creative Commons Attribution License, which permits unrestricted use, distribution, and reproduction in any medium, provided the original work is properly cited.

Essentially, financial development is a continuous and time-varying process. This paper explicitly accounts for this by introducing functional data analysis to convert discrete observations into a smooth curve in order to represent the continuous process of financial development at the regional level. Regional disparities in financial development in China are reexamined from three dimensions: financial scale enlargement, financial structure adjustment, and financial intermediary efficiency. Disparities are then decomposed using an extended functional Theil index. It is necessary to introduce functional data into the research of financial development level. Through the functional research of China's financial development level index of three different dimensions, the financial development level can be studied at the level of speed and acceleration, which fills up the research gap. The results imply that (1) the disparity in the level of financial scale enlargement and disparity in velocity and acceleration of financial intermediary efficiency are both statistically significant across regions, while the regional disparity in financial structure adjustment is insignificant, and (2) the downward trends of the total disparity in three dimensions are all dominated by the declining intraregional disparities. Since all 31 provinces have broken the traditional regional division, China does not need to implement region-specific protective policies in strengthening its deepening financial reforms.

\section{Introduction}

With rapid economic growth following the 1978 opening-up policy, regional financial disparity has become a major challenge in China [1]. In developing countries, economic development leads to certain regional disparity which is normal $[2,3]$, and the disequilibrium approach to financial development is considered a successful outcome of China's economic reform policy [4]. However, long-standing and increasing regional disparities not only negatively affect financial resource allocation and economic growth but also endanger society's welfare and even political stability. This has attracted much attention from academics $[5,6]$ and the Chinese government.

1.1. Literature Review. Starting from the study of financial development by economist Patrick $\mathrm{H} \mathrm{T}$ in his thesis Financial Development and Economic Growth in
Underdeveloped Countries in 1966, the theory of financial development has attracted more and more attention in academia. In 1969, Raymond Goldsmith conducted a quantitative analysis on the concept of financial structure in his book Financial Structure and Financial Development, which laid the foundation for the development of financial theory [7]. In China, Wu divided financial development research into four aspects, financial assets, institutions, markets, and systems, in his book China's Financial Development Theory [8]. Levine used the current liabilities of financial intermediaries, the proportion of commercial banks and central banks in the total credit balance, and the scale of credit to private and public systems through the banking system [9]. Zhang proposed the concept of "financial growth." [10] Yin and Xiao focused on the level of financial development [11]. Zhang and Yuan deepened the concept of financial growth [12]. Zhang and Pan combined the development of financial intermediaries with the evaluation system of the security market to measure financial 
development. Yang and Zhu focused on the promotion of financial development in the central region of my country on the regional economy [13]. Zhou and Wang used financial related ratios to measure the level of my country's interregional financial development [14]. Hu analyzed the financial development process of various regions in my country through the relevant data of financial structure deposits and loans [15]. Li used the ratio of non-state-owned enterprise loans to GDP in the bank data of various regions of our country to measure the financial development of different regions of our country [16]. Saciand set indicators from two aspects, banks and stock markets, to measure the level of financial development. Guan analyzed the financial development level of central cities mainly from two aspects: banking and insurance. Dou and Wang used the total amount of deposits and loans per capita in each region of my country to compare the differences in financial development in different regions and absolutized the analysis results, using the ratio of the deposit and loan balances of financial institutions in each region to GDP to compare different regions' differences in financial development and relative analysis [17]. Ye analyzed the level of my country's regional financial development based on the two perspectives of financial breadth and financial depth [18].

The definition of the concept of financial development is a constantly changing process. Due to the differences in the specific environment, academic background, and research perspectives of different scholars, there are certain differences in the cognition of the nature and scope of financial development. The understanding of the company will inevitably go through a process from shallow to deep, but there is no comprehensive and systematic analysis of the financial development theory, and there are still imperfections.

The analysis of regional differences in China's financial development has also attracted the attention of some scholars in recent years. Zeng pointed out that my country has a vast territory, a large population, and uneven economic development, especially since the reform and opening up, the gap in regional economic development has gradually widened. Coastal and inland areas, special and nonspecial zones, and cities and rural areas have shown different economic developments. And how to analyse development trends is necessary to specialize in research, in which special attention should be paid to the study of regional finance [19]. Zhang adopted the "neoclassical equilibrium hypothesis," "circular accumulation causality principle," and "Williamson's inverted-U hypothesis" [20]; after making a comparative analysis, it is believed that regional differences in the economic structure will inevitably lead to regional differences in financial system arrangements and financial structures and expound the regional convergence of the financial structure, and regional finance presents a similar economic structure. Zhang [21], Lu and Li [22], and Jin and Tian [23] all discussed the regional differences in China's financial development. Zhao and Ma studied the differences in China's regional financial growth based on the Theil index. The results show that the differences in regional financial growth in my country are mainly derived from the differences in financial growth between regions, and the differences in regional financial growth only constitute a small part of the overall difference in financial growth. Among the contributions to the overall difference, the interregional contribution is much greater than the intraregional contribution; the eastern part of the region has a greater contribution to the overall difference, while the central and western regions have a small contribution to the overall difference [24].

The following also attempts to use the Theil index to study the regional differences in China's financial development, but the method of calculating the Theil index, the time period of the study, and the division of economic regions are different from those of Zhao and $\mathrm{Ma}$, and their conclusions are quite different.

1.2. Significance. The approaches to investigate regional disparities range from simple measurements such as coefficient of variation and Gini index [25] to more sophisticated techniques such as Theil index and Williamson coefficient [26]. Despite the success of the existing approaches in measuring financial disparity, some questions remain unanswered. This paper adopts functional data analysis (FDA) to quantitatively examine regional disparities from both static level and multiple dynamics perspectives, with the following significance.

(1) Most of the previous studies show that the static level of regional differences in financial development is statistically significant. Using the FDA's research methods in this article, we can obtain whether the dynamic differences in speed and acceleration are also significant.

(2) China's financial system has changed from a single banking system to a multitiered system [27]. How to construct an evaluation system for the level of financial development has become the top priority for studying the level of financial development. This paper clarifies the concept of financial development and constructs a comprehensive financial development indicator system to better reflect the level of financial development in China and various regions.

(3) In the study of regional differences in the level of financial development, there are not many studies using the Theil index to decompose regional disparities, and the conclusions on which of the disparities between groups and within groups are dominant are not uniform. This paper uses the innovative functional Theil index decomposition research method to decompose the regional disparities in the financial development level and obtain the decisive factors that cause regional differences in financial development.

(4) According to the research of this article, the disparities in the level of regional financial development are dominated by the disparities between groups, so it is concluded that the government does not need to implement financial development protection policies for specific regions, and the government provides a basis for the formulation of policies to promote regional financial development. 
Although the data are discrete, we assume continuous financial development over time. The smooth curve from FDA is used to compare patterns and variability of financial development over time and across regions. Specifically, dynamic insights can be mined from the curves' slopes and curvatures. As a big data analysis method, FDA has been widely used [28]. However, this is the first time it has been applied to examine regional financial disparities. Thus, our findings are expected to provide further insights on China's financial system reform.

\section{Data and Methods}

Total financial assets include broad money M2, securities, and insurance funds [29]. China's share of stocks, securities, and insurance in total financial assets is growing fast [30]. To comprehensively measure financial development, especially changes in financial assets' composition, we extend the concept of financial development into three dimensions. Financial scale enlargement (FSE) is total financial assets divided by GDP, reflecting the share of financial assets in regional wealth [31]. Financial structure adjustment (FSA) is the ratio of total security assets to total financial assets, reflecting changes in the indirect financing size [32]. Financial intermediary efficiency (FIE) is the loan balance of financial institutions divided by their savings balance, measuring financial intermediaries' financing ability [33]. In the current research on regional differences in China, some researchers use large areas as the research base point for grouping research (such as eastern, central, and western regions), and some use urban agglomerations as the research base point for grouping research [34] (such as the Yangtze River Delta and Pearl River Delta). Thinking about the issue of data availability, this thesis takes the three major regions of China as the research base. A panel dataset of 31 provinces across 3 regions including the east region (including Beijing, Tianjin, Hebei, Liaoning, Shanghai, Jiangsu, Zhejiang, Fujian, Shandong, Guangdong, and Hainan), central region (including Shanxi, Inner Mongolia, Jilin, Heilongjiang, Anhui, Jiangxi, Henan, Hubei, Hunan, and Guangxi), and west region (Sichuan, Guizhou, Yunnan, Xizang, Shaanxi, Gansu, Qinghai, Ningxia, and Xinjiang) which covers the period from 2006 to 2018 is employed for empirical analysis. The annual data are obtained from the Almanac of China's Finance and Banking (2006-2018).

2.1. Methods' Overview. In traditional discrete data statistical methods, discrete data can reflect the general trend and degree of dispersion of the data as a whole and can describe the general characteristics of the absolute level of the data to a certain extent, which has certain advantages and ease of operation, but at the same time, we should note that traditional statistical methods have certain shortcomings. For example, the trajectory of data changes cannot be reflected in real time; in the case of missing data in a specific year, the next step of statistical analysis cannot be performed; it is impossible to study the statistical characteristics of the speed and acceleration of the data change during the data change, so it is impossible to study the influencing factors behind the data change and the change in the efficiency of the data itself. As a big data analysis method, functional data analysis (FDA) solves some of the problems in traditional data statistical analysis. FDA first uses the nonparametric fitting method of basis function expansion to reconstruct the discrete observation data into continuous eigenfunctions. In order to eliminate the noise effect polluted by the measurement error and highlight the dominant trend of the eigenfunction, we use the roughness penalty method to smooth the function curve, which not only reduces the interference of the error but also further highlights the trend of its own change and its own fluctuation law. This allows discrete statistical data to be processed into a continuous curve that eliminates noise and highlights the dominant trend through functional processing and can more intuitively judge the trajectory of the data, the degree of dispersion, and the convergence and divergence of data in different regions. At the same time, FDA does not force all samples to be taken with the same time point and interval in the form of regular sampling and releases the structural restriction and distribution hypothesis for data collection, so as to be more flexible and comprehensive in describing the actual problem [35]. Especially, it is available for transactional analysis against the dynamic difference of the related variables based on the multiple-order derivative of the eigenfunction when the eigenfunction is reconstructed based on the discrete static observation data so as to figure out the changes of the intrinsic connection among variables and explore the fluctuating trend and dynamic characteristics of the changing trend in real life.

Variance analysis is used in many studies and has many advantages. Variance analysis is not limited by the number of comparison groups. By analyzing the contribution of different sources of variation to the total variation, the influence of controllable factors on the research results can be determined. At the same time, compared with extreme value analysis and maximum value analysis, variance analysis can describe the differences between data more finely. This article mainly uses functional variance analysis to test the significance of the differences in regional financial development levels. Similar to the variance analysis of discrete data, the functional variance analysis is mainly used to compare multiple groups of function sets to find out whether they are independently and identically distributed; besides, the comparison object is the expectation of each function set [36].

The traditional Theil index method, while studying the disparities, can decompose the differences in grouped data, so as to analyze whether the dominant factor causing the differences in grouped data is between-group factors or within-group factors. The traditional Theil index method provides an excellent research method for studying the disparities in China's regional financial development. Further research can determine whether the dominant factor causing the disparities in China's regional financial development is the disparity between regions or within regions and formulate balanced finance for the government development policy provides research basis, but at the same time, 
we noticed that the traditional Theil index method also has the limitations of traditional statistical methods: it can only decompose regional disparities at a specific point in time. In this paper, the innovative functional Theil index decomposition method can continuously decompose our regional disparities in a continuous time, so as to obtain more excellent disparity decomposition results. Therefore, the functional Theil index provides a dynamic and continuous representation of the regional imbalance in the heterogeneous regional structure.

2.2. Smoothing with Roughness Penalty. As a big data analysis method, functional data analysis (FDA) is widely used in the potential trajectory identification of actual observation data provided that each sample is an independent realization of the potential random process in the Hilbert space [37]. The primary task of FDA is to reconstruct the continuous eigenfunction based on the discrete observation data and using nonparametric fitting expanded by the basis function. In view of the actual condition of the specific problem, the observation value always suffers from the disturbance due to different sample intervals and observation errors, while within the FDA frame, the discrete data, such as time series and longitudinal data, will be converted into the continuous function called curve data. The first step in FDA is to convert the discrete data into a smooth curve or function to represent the continuous process of financial development. A common method to represent a priori unknown function $x_{i}(t)(i=1, \ldots, n)$ from observations $y_{i 1}, \ldots, y_{i T_{i}}$ is to express it as a sum of analytically known basis functions $\phi_{1}(t), \ldots, \phi_{K_{x}}(t)$, that is, $x_{i}(t)=\sum_{k=1}^{K_{x}} \beta_{i, k} \phi_{k}(t)$. It could be expressed in the form of the linear combination of the basis function for its eigenfunction through the minimization of the residual sum of squares using FDA.

$$
\mathrm{SSE}=\sum_{j=1}^{T_{i}}\left[y_{i j}-y_{i}\left(t_{i j}\right)\right]^{2}=\sum_{j=1}^{T_{i}}\left[y_{i j}-\sum_{k=1}^{K} \zeta_{i k} \phi_{k}\left(t_{i j}\right)\right]^{2},
$$

where $\varphi_{k}$ refers to the basis function, $K$ refers to the maximum number of the required basis functions, and $\xi_{k}$ refers to the coefficient of the basis function.

To eliminate the measurement error and highlight the dominant trend of $x_{i}(t)$, the roughness penalty approach is employed for smoothing the functional curve. Ramsay et al. proposed the concept of roughness penalty and defined it as the integral sum of the second derivative $y_{t}^{\prime \prime}(t)$. Assume that $\Phi(t)=\left\{\phi_{1}(t), \ldots, \phi_{L}(t)\right\}$ is the optimal basis function in the Hilbert space; then, the fitting residual sum of squares for roughness penalty against smoothing $\operatorname{PENSSE}_{\kappa}$ is

$$
\operatorname{PENSSE}(\lambda)=\sum_{j=1}^{T_{i}}\left[y_{i j}-y_{i}\left(t_{i j}\right)\right]^{2}+\lambda \int_{T}\left[y_{i}^{\prime \prime}(t)\right]^{2} \mathrm{~d} t
$$

The eigenfunction $y_{i}(t)$ in equation (2) presents linear approximation from the basis function under the standard of the minimum penalty residual sum of squares, that is, $y_{i}(t)=\sum_{l=1}^{L} \beta_{i l} \varphi_{l}(t)$; the smoothing parameter value $\lambda$ is used to weigh the proportional relation between the model fitting goodness and the smoothing degree of function curve. The optimal value will be determined with the standard of the minimum generalized cross-validation $\operatorname{GCV}(\lambda)$, i.e.,

$$
\operatorname{GCV}(\lambda)=\left(\frac{T_{n}}{T_{n}-d f(\lambda)}\right)\left(\frac{\operatorname{PENSSE}_{\lambda}}{T_{n}-d f(\lambda)}\right)
$$

$d f(\lambda)$ in equation (3) represents the trace of the projection matrix $S_{\Phi, \lambda}=\Phi\left(\Phi^{\prime} \Phi+\lambda \Phi\right)^{-1} \Phi^{\prime}$, i.e., $d f(\lambda)=$ trace $S_{\Phi, \lambda}$, where $\mathbf{R}=\int D^{2} \phi(s) \cdot D^{2} \phi^{\prime}(s) \mathrm{d} s$ represents the matrix composed of outer product integration of the basis function's second derivative. $T_{n}$ refers to the sample size of the time point. Therefore, to minimize equation (2), the optimal solution is $\widehat{\beta}=\left(\Phi^{\prime} \Phi+\lambda R\right)^{-1} \Phi^{\prime} y$, where $\lambda$ makes GCV reach its minimum value. However, the number $K$ of the selected basis function will be assumed to be optimal, which should act as the precondition when $\operatorname{GCV}(\lambda)$ obtained from equation (3) is used to determine the optimal value of $\lambda$. Under normal conditions, due to the fact that a relatively large number of basis functions $K$ may lead to overfitting or underfitting of data in most cases, for relatively discrete data, a relatively small number of basis functions $K$ are beneficial for the fitting performance compared with the relatively large ones $K$ [38]. Taking into account the smoothness of $x_{i}(t)$, defined by the integrated square of the second derivatives $D^{2} x_{i}(t)$, the coefficients $\beta_{i, k}, k=1, \ldots, K_{x_{i}}$, are estimated by minimizing the penalized residual sum of squares (PENSSE):

$$
\operatorname{PENSSE}\left(\lambda_{x}, K_{x}\right)=\sum_{j=1}^{T_{i}}\left(y_{i j}-\sum_{k=1}^{K_{x}} \beta_{i, k} \phi_{k}\left(t_{j}\right)\right)^{2}+\lambda_{x} \int_{\mathrm{T}}\left[D^{2} x_{i}(t)\right]^{2} \mathrm{~d} t,
$$

where $\lambda_{x}$ in equation (4) specifies the weighing between the least-squares approximation and the roughness. The parameter $\lambda_{x_{i}}$ specifies a tradeoff between the least squares fitting and the roughness of $x_{i}(t)$. Larger values of $\lambda$ will increase smoothness. The pairs of $\left(\lambda_{x}, K_{x}\right)$ giving the smallest value of the generalized cross-validation (GCV) will be the optimal smoothing parameters [36]. Therefore, GCV of the unit is extended into the function of two variables in this paper, so as to identify the optimal value of $\lambda$ and $K$ in the meantime. The criterion after extension is

$$
\operatorname{GCV}(\lambda, K)=\left(\frac{T_{n}}{T_{n}-d f(\lambda, K)}\right)\left(\frac{\operatorname{PENSSE}(\lambda, K)}{T_{n}-d f(\lambda, K)}\right)
$$

It is possible to obtain the corresponding value of $\lambda$ and $K$ for the optimal fitting effect, so as to mitigate the disturbance brought by the error, and further highlight its changing trend and fluctuating principle.

2.3. Functional Variance Analysis. Similar to the variance analysis of discrete data, the functional variance analysis is mainly used to compare multiple groups of function sets to 
find out whether they are independently and identically distributed; besides, the comparison object is the expectation of each function set [36]. As per the variance analysis, it is deemed that the difference of the expectation of various group data is mainly from two sources: one is the error related to the intrinsic reason of packet processing, which contains the information reflecting the difference of different groups, called "intergroup error"; besides, it is expressed with the sum of squares for the difference between the average value of each group and the total average value, written as SSR, and the degree of freedom is $g-1$ (assume that there are $g$ groups); the other is the random error caused by external factors, such as observation measures and environment, called inner-group error, and it is expressed with the sum of squares for the difference between the innergroup observation value and the average value of each group, written as SSE, and the degree of freedom is $n-g$; assume there are $n$ observation values in total. Written as $\mathrm{MSR}=(\mathrm{SSR} / g-1)$ and MSE $=(\mathrm{SSE} / n-g)$, if $(\mathrm{MSR} / \mathrm{MSE})$ $\approx 1$, it is deemed that the data of each group come from the united totality, while in the case that MSR $\gg$ MSE, it is deemed that the samples in different groups may come from different totalities. When extending to the functional field, it could be obtained that $g$ is written as the number of groups, and $f_{i j}\left(i=1, \ldots, g ; j=1, \ldots, n_{i}\right)$ refers to the $j$ function of the $i$ group; therefore, the $F$ statistical magnitude of the functional variance analysis is

$$
F=\frac{\sum_{i=1}^{g}\left(n_{i}\left|\overline{f_{i}}-\bar{f}\right|^{2} /(g-1)\right)}{\sum_{i, j}\left(\left|f_{i j}-\overline{f_{i}}\right|^{2} /(n-g)\right)},
$$

where $\quad \overline{f_{i}}=\left(\overline{f_{i}}\left(t_{1}\right), \ldots, \overline{f_{i}}\left(t_{T}\right)\right)^{\prime}$ and $\bar{f}=\left(\bar{f}\left(t_{1}\right), \ldots, \bar{f}\right.$ $\left.\left(t_{T}\right)\right)^{\prime}$ are the functional expectations of the group and the totality, respectively, $\overline{f_{i}}(t)=\sum_{j}^{n_{i}}\left(f_{i j}(t) / n_{i}\right)$, and $\bar{f}(t)=\sum_{i=1}^{g}$ $\sum_{j=1}^{n_{i}}\left(f_{i j}(t) / n\right), n=\sum_{i=1}^{g} n_{i}$. Given the original hypothesis $H_{0}: \overline{f_{1}}=\cdots=\overline{f_{g}}$, the critical value $P_{H_{0}}\left\{F_{n}>F_{n, \alpha}\right\}=\alpha$ is calculated at the significance level $\alpha$. The size of $F_{n}, F_{n, \alpha}$ is compared to complete the verification of this hypothesis [39].

2.4. Functional Theil Index. The standard Theil entropy index [40] is extended to FDA and used to decompose the dynamics of the overall financial disparity into intraregional and interregional components $[7,8,41-46]$. Using a province as the regional unit, let $g(1, \ldots, G)$ indexes be the number of regions, with $n_{g}$ provinces in region $g$. Taken the dimension of FSE $(t)$ as an example, total disparity between provinces $T(t)$ can be calculated as

$$
T(t)=\sum_{g=1}^{G} \sum_{i=1}^{n_{g}}\left(\frac{\mathrm{FSE}_{g i}(t)}{\mathrm{FSE}(t)}\right) \ln \left(\frac{\left(\mathrm{FSE}_{g i}(t) / \mathrm{FSE}(t)\right)}{(1 / N)}\right),
$$

where $\mathrm{FSE}_{g i}(t)$ is the level of financial scale enlargement for a province and $\operatorname{FSE}_{g}(t)=\sum_{i=1}^{n_{g}} \operatorname{FSE}_{g i}(t)$ and $\operatorname{FSE}(t)=\sum_{g=1}^{G}$ $\sum_{i=1}^{n_{g}} \operatorname{FSE}_{g i}(t)$ are the overall scales for each region and all provinces, respectively. $N=\sum_{g=1}^{G} n_{g}$ denotes the number of provinces. The disparity among provinces of each region $T_{g}(t)$ is defined similarly: $T_{g}(t)=\sum_{i=} 1^{n_{g}}\left[\mathrm{FSE}_{g i}(t) / \mathrm{FSE}_{g}\right.$ $(t)] \ln \left[\left(\mathrm{FSE}_{g i}(t) / F S E_{g}(t)\right) /\left(1 / n_{g}\right)\right]$.

Using the functional Theil index decomposition, financial development disparities are separated into two components: the intraregional disparity $T_{W}(t)$ and interregional disparity $T_{B}(t)$ :

$$
\begin{aligned}
T(t)= & \sum_{g=1}^{G}\left(\frac{\operatorname{FSE}_{g}(t)}{\operatorname{FSE}(t)}\right) T_{g}(t) \\
& +\sum_{g=1}^{G}\left(\frac{\operatorname{FSE}_{g}(t)}{\operatorname{FSE}(t)}\right) \ln \left(\frac{\left(\operatorname{FSE}_{g}(t) / \operatorname{FSE}(t)\right)}{\left(n_{g} / N\right)}\right) \\
= & T_{W}(t)+T_{B}(t),
\end{aligned}
$$

where $T_{W}(t)$ is the weighted average of the interprovince financial development disparity for each region $T_{g}(t)$. Noticeably, every part in equation (8) is functional; the relative importance of $T_{W}(t)$ and $T_{B}(t)$ on $T(t)$ can be continuously identified.

\section{Results and Discussion}

3.1. Data Smoothness and Significance Tests. By minimizing the objective function $\operatorname{GCV}(\lambda, K),\left(\lambda=10^{-1.65}, K=5\right)$ is identified as the optimal parameter for smoothing FSE. The other two pairs of optimal penalty parameters for smoothing FSA and FIE are similarly determined as $\left(\lambda=10^{-3.85}, K=5\right)$ and $\left(\lambda=10^{-4.3}, K=12\right)$, respectively. The three dimensions are then smoothed at the level of these three penalty parameter pairs. Figure 1 presents the descriptive statistics for the financial development functional form. Figure 1(a) shows that, despite the fluctuations in some years, a substantial increase in the three dimensions is the common feature of China's financial development. In contrast, the three trajectories' standard deviation functions demonstrate a declining trend, indicating that the financial disparity across 31 provinces in China is dwindling.

China is divided into three regions: eastern, central, and western [47]. To test the significance of the financial disparity within regions, we conducted a functional ANOVA with 100 bootstrap resamples on both absolute levels and growth dynamics.

3.1.1. Levels of Regional FSE. As per the test result of FSE, the regional disparity of FSE is significant. It can be seen from Figure 2 that the FSE levels of all the three regions are maintained at the upward trend within the period from 2006 to 2018, which indicates that the financial development in the eastern, central, and western region of China has maintained an upward trend in recent years.

By horizontal comparison, it can be seen that the FSE level of the eastern region is taking the lead among the rest regions in China from 2006 to 2018, and this is due to the nonbalanced development strategy of "step-by-step development" implemented by the Chinese government. When the strategy of "Develop East Region First" [48] is 


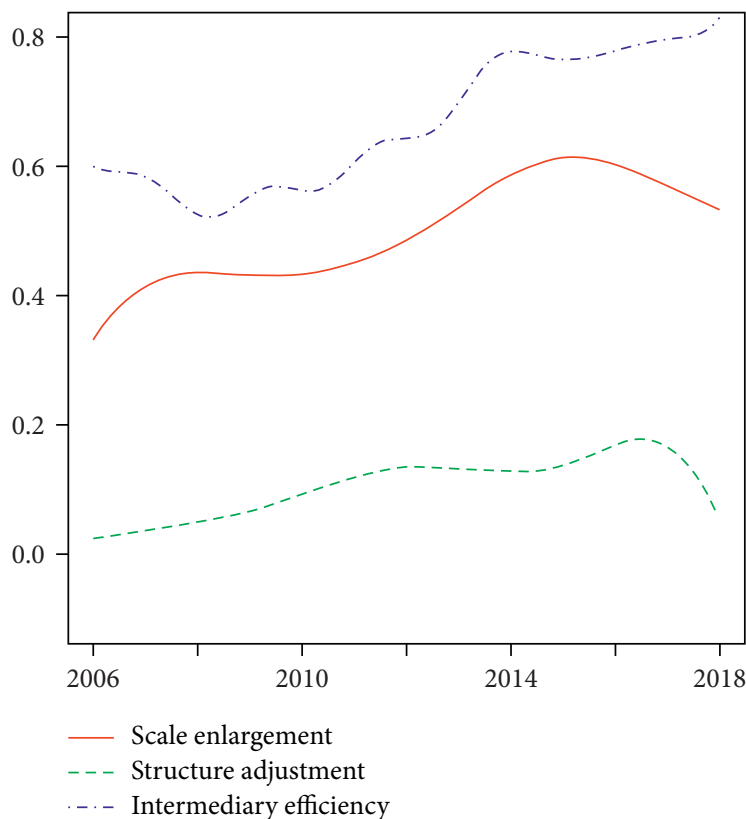

(a)

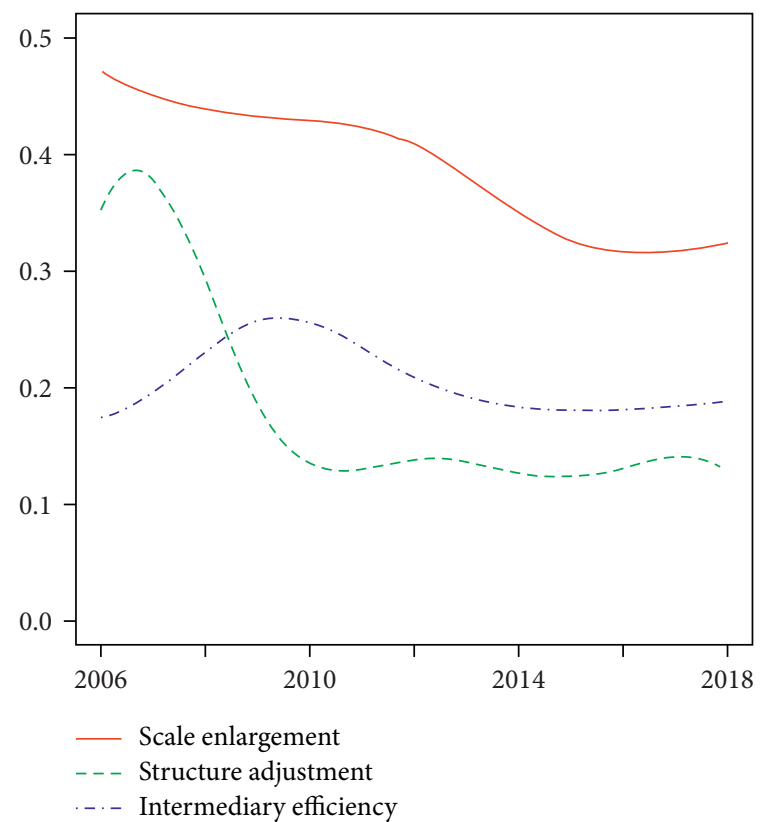

(b)

FIgURE 1: Statistical description of financial development in China. (a) Functional means. (b) Standard deviation functions.

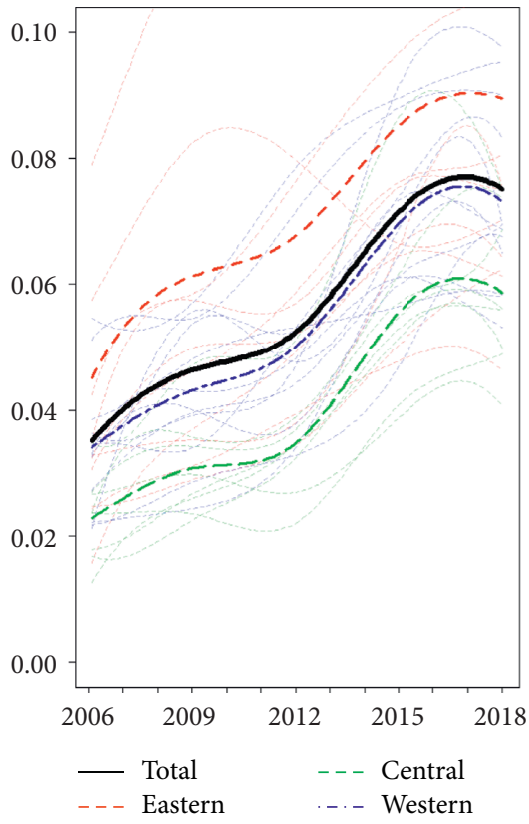

(a)

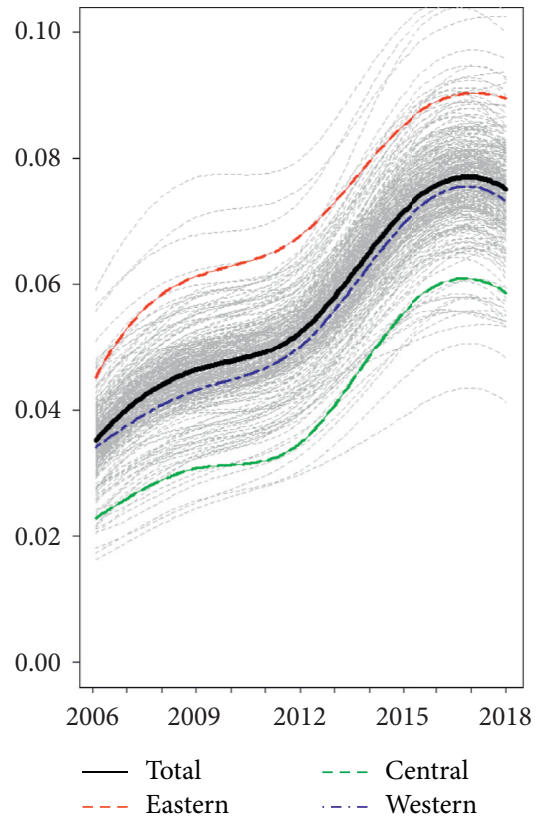

(b)

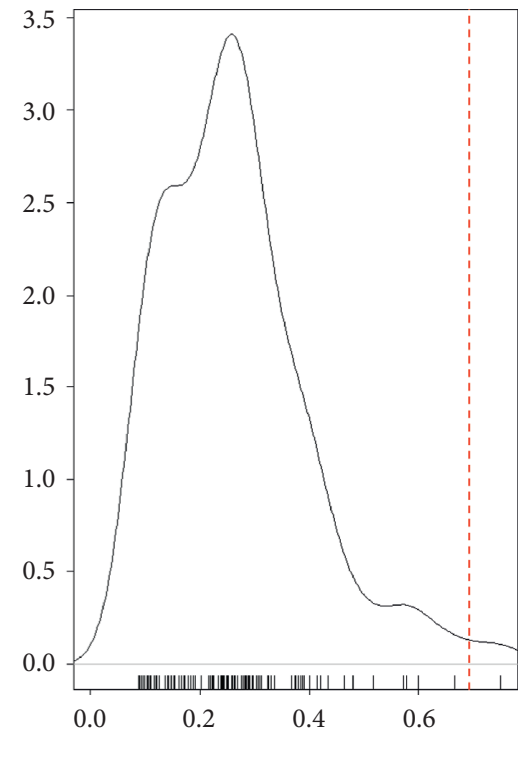

(c)

Figure 2: FANOVA test for the regional disparity in the absolute level of FSE. (a) Functional mean. (b) Bootstrap resamples, $n=100$. (c) Density plot for bootstrap resamples; $N$ levels: 3 , stat $=0.692$, and $p$ value $=0.01$.

proposed, the eastern regions are supported by the state policies and enjoy the favorable policy for the financial development. In the meantime, the eastern regions take the huge advantages over other regions, including geographical location, transportation system, talent population, and industrial structure; the disparity in terms of the FSE level compared with the central and the western regions is thus enlarged. The FSE level in the central regions is always relatively low, which is lower than that of other regions for a long term. The FSE level of the western regions is always between the eastern region and the central region from 2006 to 2018 . This is mainly because that, with the deepening of "the great western development strategy" of China, a large amount of supportive 
policies and capital transfer have been given to the western region by the country, which promote the economy development in the western region and further improve the financial development level [49].

By combining Figures 3 and 4, it can be seen that although there is big disparity at the absolute level in the contribution to the regional wealth due to the financial development, no significant difference is seen in the velocity and acceleration for the increase of the FSE level in each region. This means that each region has realized the importance of the financial development for its promotion to the economy; and it is actively adjusting its financial structure, introducing talents [50], creating the financing environment [51], and perfecting the financial law [52], thus improving the financial development to closing the regional gap. Therefore, there is no significant regional disparity in terms of the growth potential of FSE.

3.1.2. Levels of Regional FSA. FSA level is available for figuring out the ratio of direct financing in the financial assets and reflecting the marketing degree of the financial structure in one region. The difference of the FSA level among the three regions in terms of statistics is not significant.

Looking into Figure 5, it can be seen from the trajectory of the FSA level in the three regions, i.e., the east, the center, and the west, that the three trajectories present an inverted "U"; besides, the positions of the peaks are close. All the three regions suffered from great dropping in terms of direct financing ratio before and after 2014. This is because that China has implemented the "Financing deleveraging policy" [53] to prevent systematic financial risks. The implementation of this policy resulted in the obvious rising of bond yield; besides, it also suppressed the bond issuing amount in the bond market, which further decreased the scale of direct financing and resulted in the great dropping in the three regions, i.e., the east, the center, and the west, in terms of the FSA level.

Through horizontal comparison, it is found that the FSE level of the eastern regions is always higher than that of the central region and the western region from 2006 to 2018. This is mainly because that the eastern region enjoys high-level development of economy and capital market [54]; besides, the local enterprises make active use of the capital market to raise fund based on their strong awareness of the capital market; the market for direct financing in the eastern region is much more developed than the central and western regions; consequently, the ratio of direct financing in the eastern region is much higher than that in the central and western regions. As for the number of listed companies, the number of listed companies in the eastern region is apparently larger than that in the central and western regions [55]; besides, the ratio of the listed companies in the eastern region is enjoying an upward trend among all the listed companies, which directly resulted in the clearly higher FSA level in the eastern region than that in the central and the western regions. Thanks to the preferential policy [56] and the advantages in terms of talents and geographic position, the financial development level in the eastern region is improved, and the financing structure is continuously optimized.
The FSA level of the central and the western region is almost the same, which is lower than that of the eastern region. In addition to the above disadvantages compared with the eastern region, the industry lagging and single structure also bring great impact on the financing structure of the central and the western region. The finance and insurance industry occupy a large ratio in the eastern region [57], while the central and western region are still in the initial stage of industrialization with relatively low economic technology level; in this case, the natural economy still occupies certain ratio, and the ratio of the tertiary industry is low. In addition to the natural policy environment, the low economy development level and industrialization level, as well as the single industrial structure in the western region, have led to the financial structure characterized by low ratio of direct financing. In the meantime, the central and western regions have masses of large-scale state-owned industrial enterprises [58], while the number of private enterprises and listed companies is relatively small; in this case, it is easier for large-scale state-owned enterprises to obtain bank credit capital [59]; besides, small-scale private companies are also in high demand of bank credit. Additionally, the implementation of the strategy of Development for Central and Western China, the continuous input of national financial capital, and the transfer payment to these regions [60] as well as the infrastructure construction with the state-owned enterprises as the main body have accelerated the industrialization progress. The capital source of the state-owned enterprises is mainly state funding and bank loan; therefore, the economy development pattern with the state-owned enterprises as the leading role will inevitably result in the fact that the financing structure in the western region mainly depends on the indirect financing.

Looking into Figures 6 and 7, it is found that there is no significant difference among the three regions in terms of the speed and acceleration of the FSA level. The speed and acceleration of the FSA level in the eastern region are almost the same as those of the whole nation, which indicates that the change of the FSA level in the eastern region is playing a leading role in the change of the level of the whole nation. The speed and acceleration of the FSA level in the central and western regions suffered from a considerable oscillation from 2006 to 2018. This may be due to the fact that the absolute amount of direct financing in the central and western regions is relatively small; therefore, even relatively small changes may result in relatively large changes in speed; in the meantime, the central and western regions are largely affected by the national policies, while the market factors can only play a small role [61]; in this case, both the changes in policy and the magnitude of implementation strength will have impact on the growth rate of direct financing [62].

3.1.3. Levels of Regional FIE. FIE level is available to evaluate the financing efficiency of financial intermediaries. The difference of the FIE level among the three regions in terms of statistics is not significant.

Looking into Figure 8, it is found that the financing efficiency in the three regions, i.e., the east, the center, and 


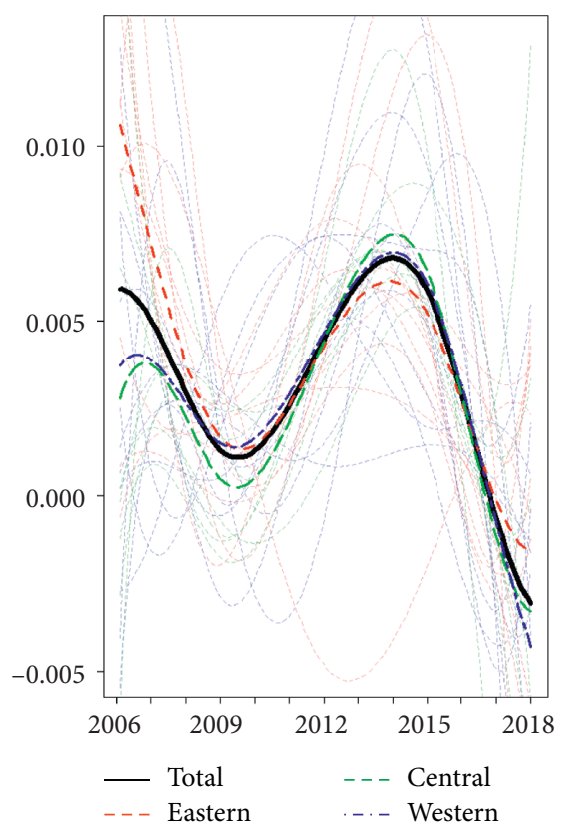

(a)

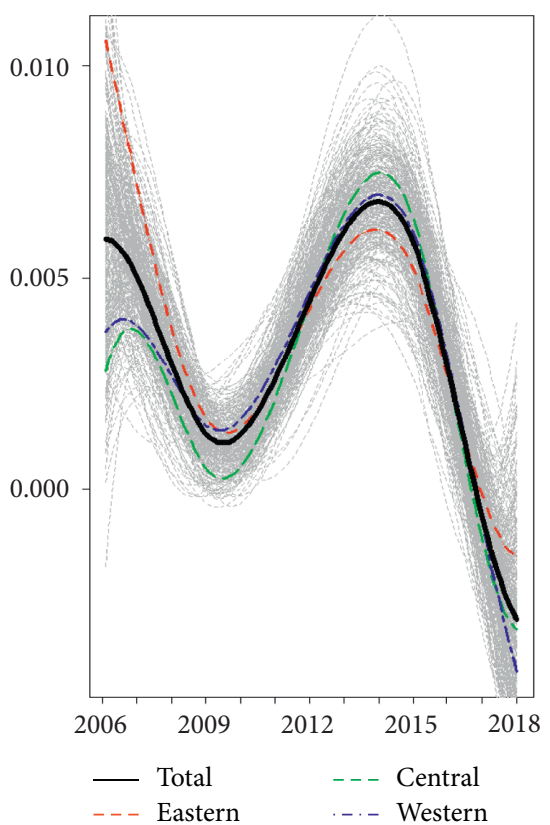

(b)

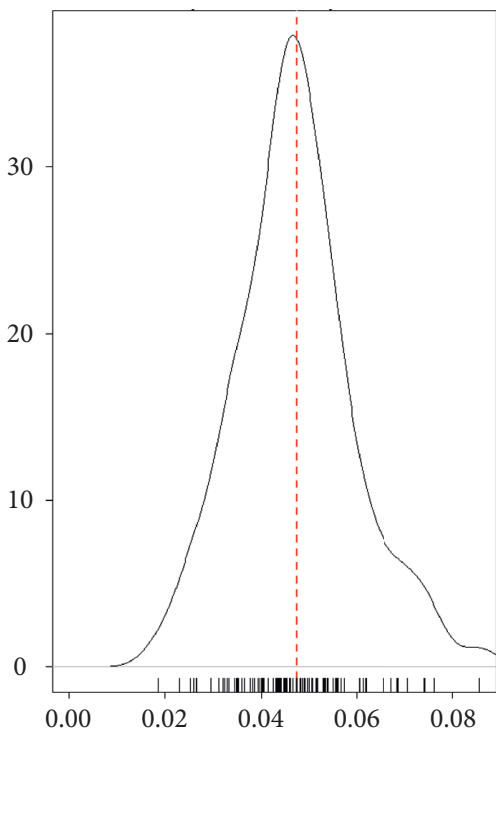

(c)

FIGURE 3: FANOVA test for the regional disparity in growth velocity of FSE. (a) Functional mean. (b) Bootstrap resamples, $n=100$. (c) Density plot for bootstrap resamples; $N$ levels: 3 , stat $=0.047$, and $p$ value $=0.46$.

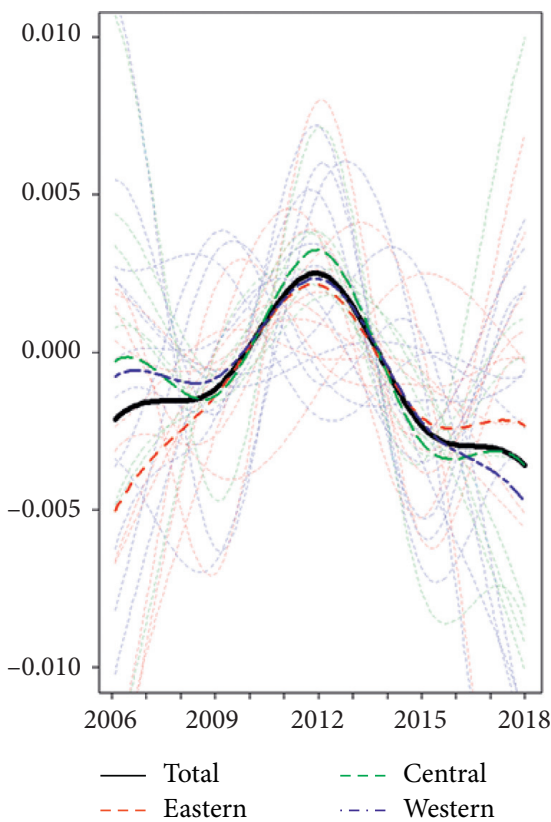

(a)

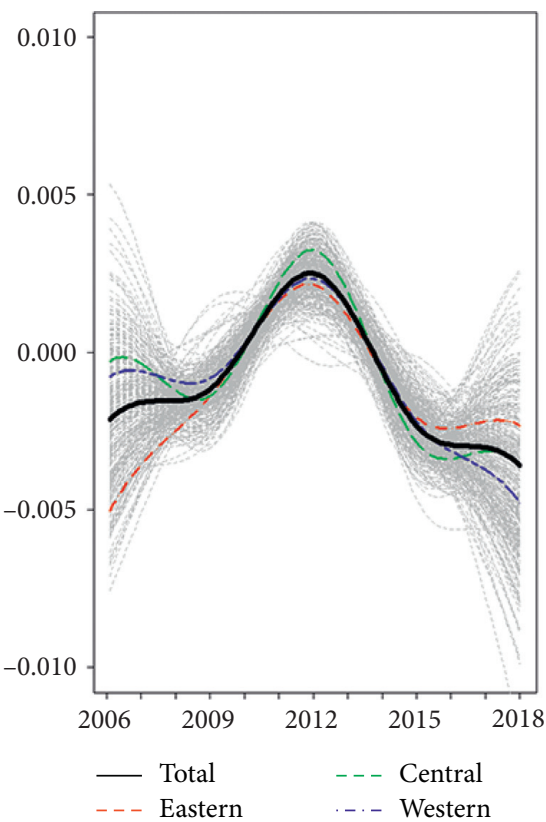

(b)

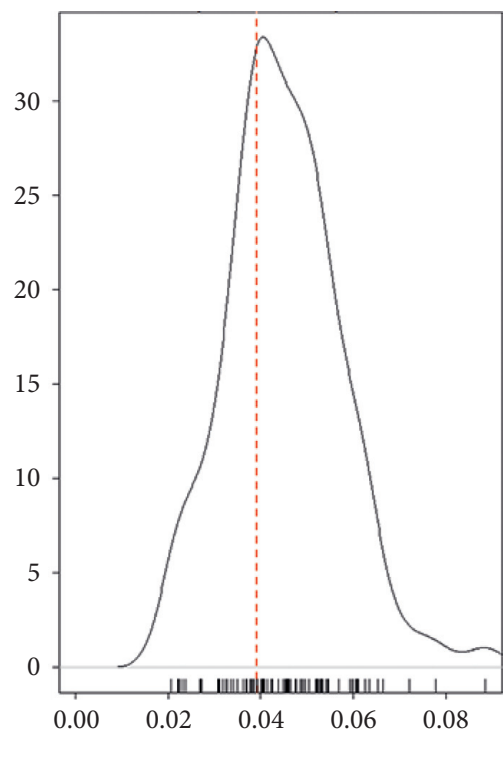

(c)

FIGURE 4: FANOVA test for the regional disparity in growth acceleration of FSE. (a) Functional mean. (b) Bootstrap resamples, $n=100$. (c) Density plot for bootstrap resamples; $N$ levels: 3 , stat $=0.039$, and $p$ value $=0.66$.

the west, has basically maintained an upward trend from 2006 to 2018. Indirect financing also suffers from the impact of the implementation of the policy of "delevering" [53], that is, the bond yield increases, which results in the declining of bond financing and the further decrease of the scale of direct financing. The enterprises have to seek for alternative financing method, so credit becomes the reliable selection of the enterprises, which has led to the further improvement of the financing efficiency of the financial institutions.

Through the horizontal comparison among the eastern, central, and western regions, it can be found that the indirect financing efficiency in the western region is the highest; this may be due to the masses of policy support obtained by the western region, the infrastructure construction in large scale, 


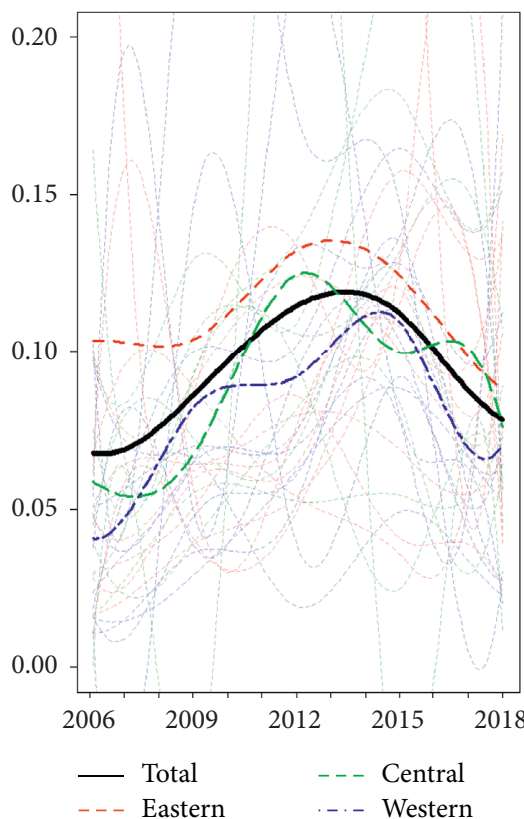

(a)

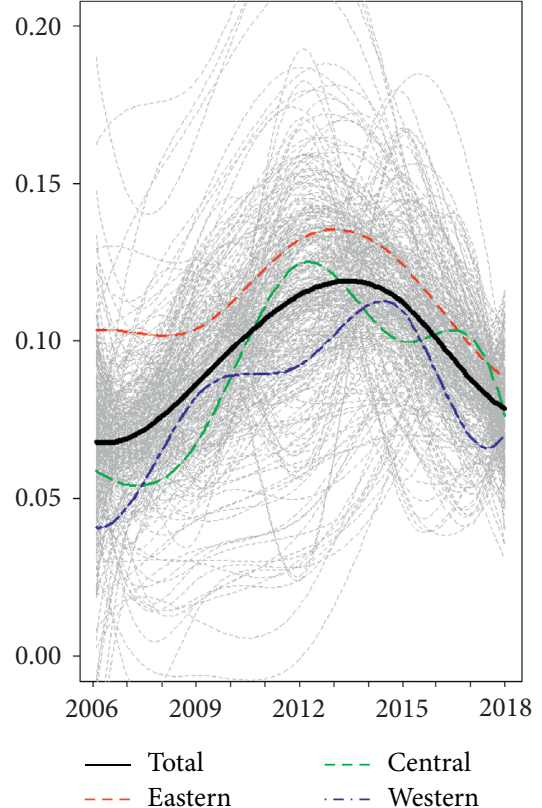

(b)

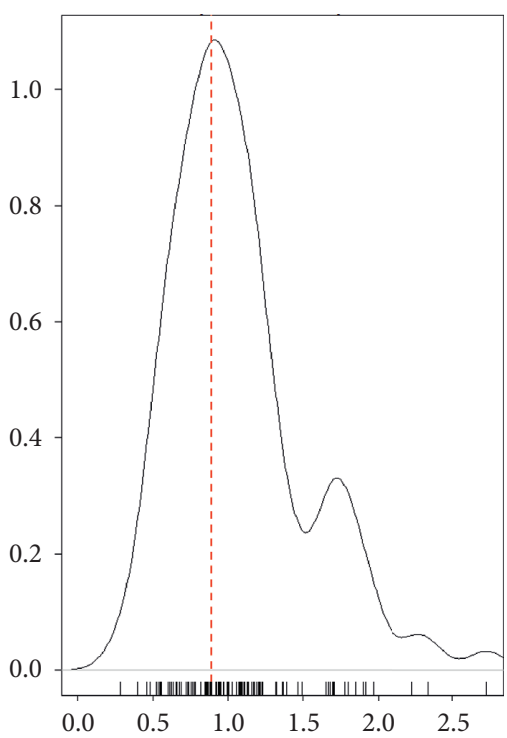

(c)

FIgURE 5: FANOVA test for the regional disparity in the absolute level of FSA. (a) Functional mean. (b) Bootstrap resamples, $n=100$. (c) Density plot for bootstrap resamples; $N$ levels: 3 , stat $=0.892$, and $p$ value $=0.58$.

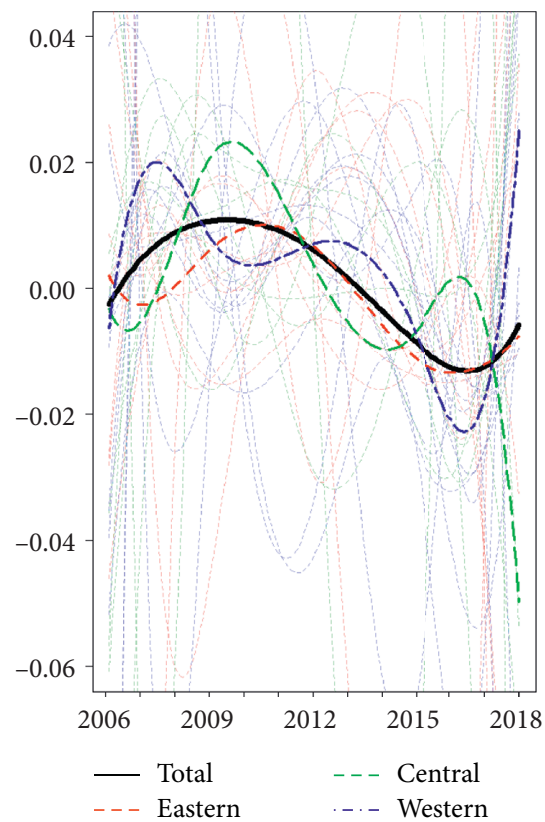

(a)

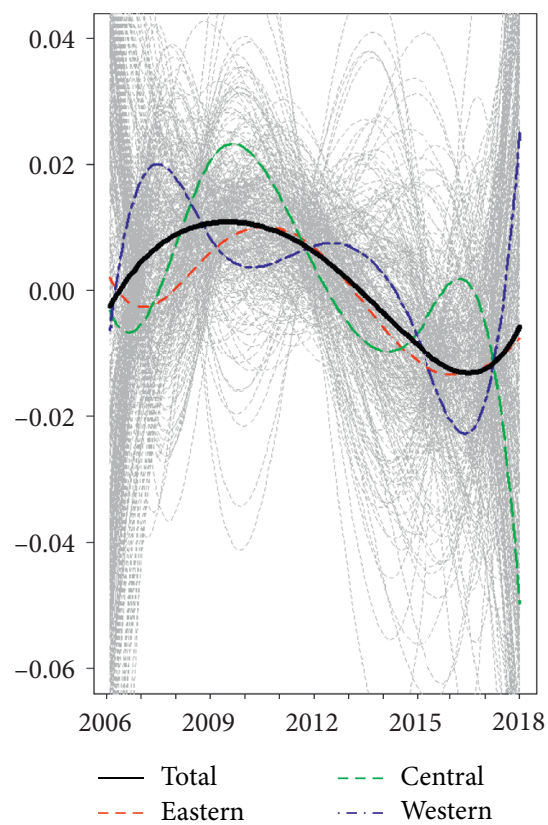

(b)

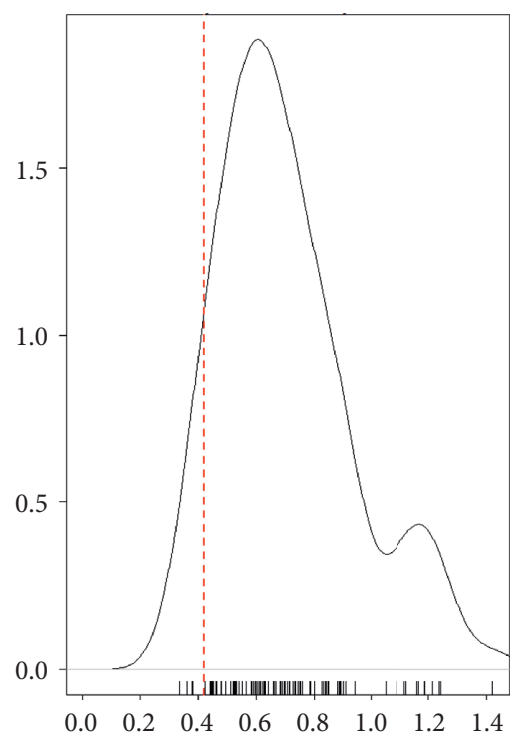

(c)

FIGURE 6: FANOVA test for the regional disparity in growth velocity of FSA. (a) Functional mean. (b) Bootstrap resamples, $n=100$. (c) Density plot for bootstrap resamples; $N$ levels: 3 , stat $=0.419$, and $p$ value $=0.95$.

and the preliminary industrialization, as well as the large amount of financial fund input by the country; state-owned enterprises that account for a large share of the western region's economy mainly finance through financial institutions, and this has greatly improved the credit scale in the western region. These procedures have improved the financing efficiency of the financial institutions in the western region $[58,59]$.
The FIE level in the eastern region is lower than that of the western region; this may be due to the fact that compared with the western region, the eastern region has the characteristics that the financial market develops in a multilevel way, and there are more financing channels; especially, direct financing occupies a certain ratio, and more alternative financial channels with higher efficiency are available 


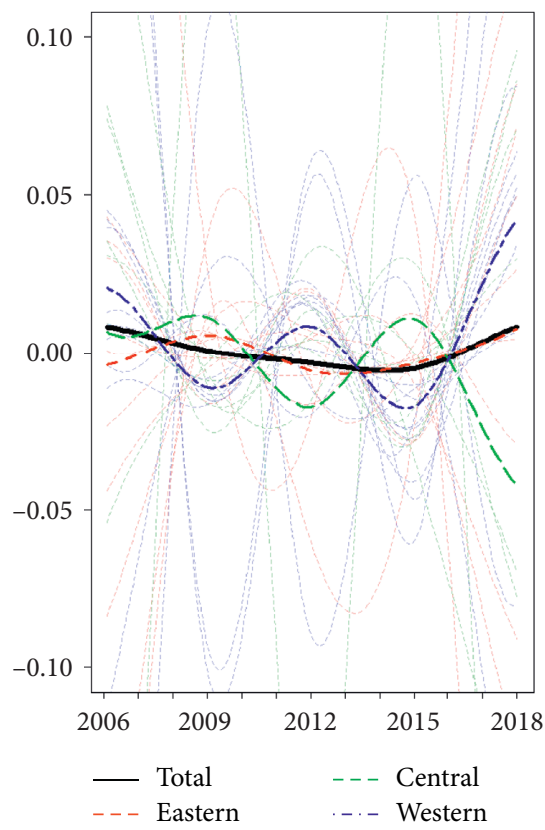

(a)

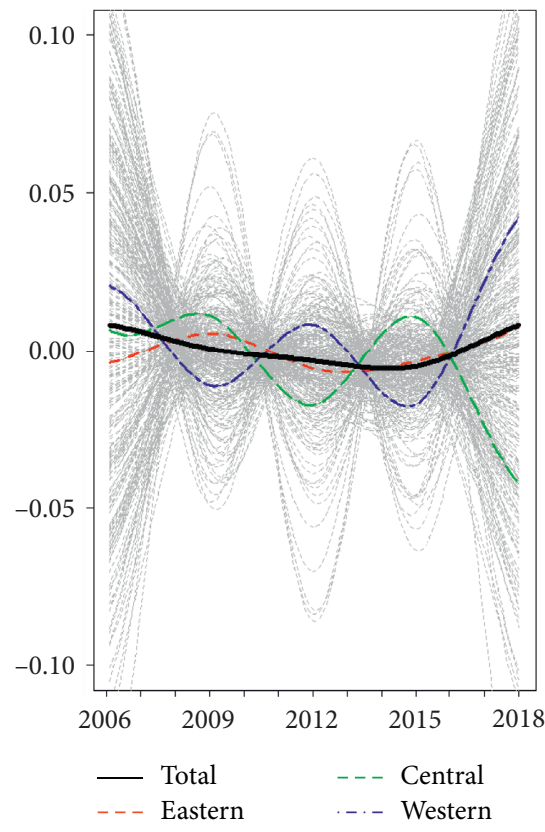

(b)

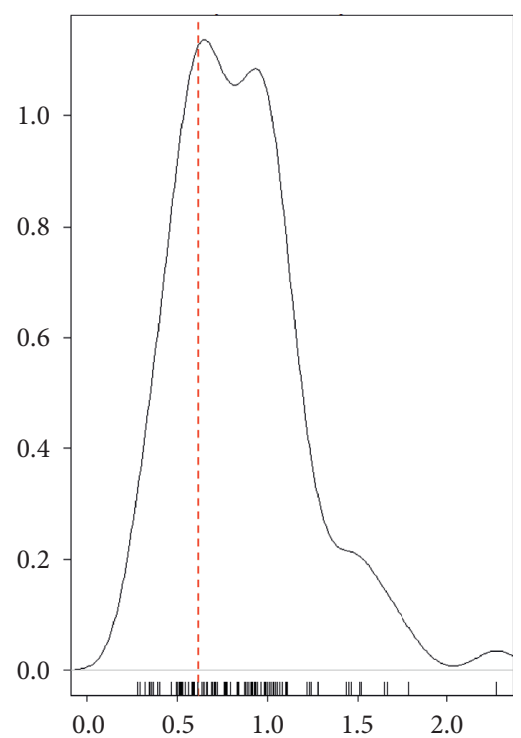

(c)

FIGURE 7: FANOVA test for the regional disparity in growth acceleration of FSA. (a) Functional mean. (b) Bootstrap resamples, $n=100$. (c) Density plot for bootstrap resamples; $N$ levels: 3 , stat $=0.62$, and $p$ value $=0.71$.

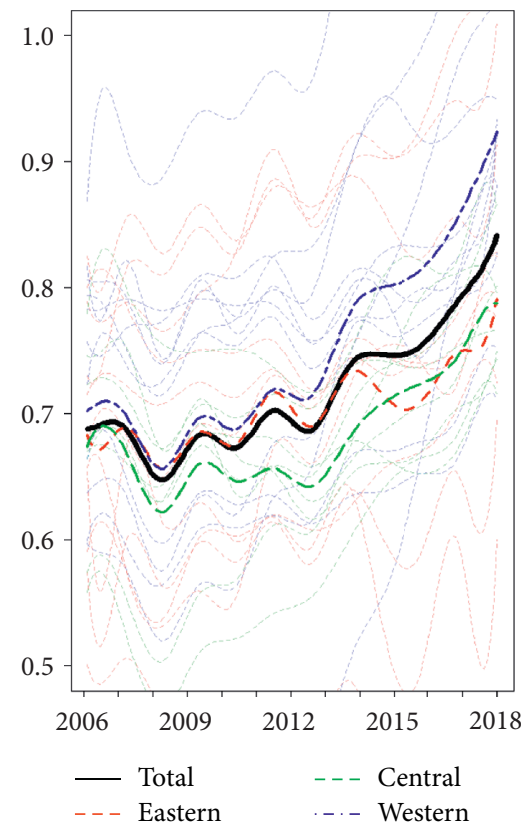

(a)

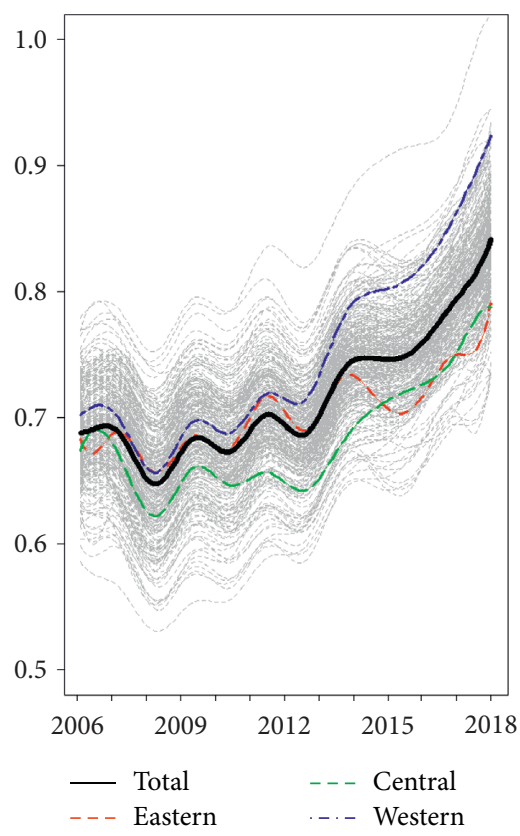

(b)

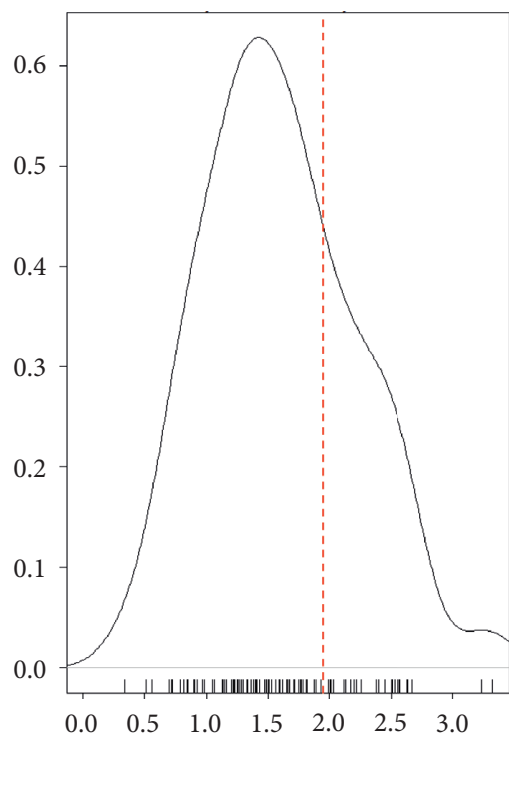

(c)

FIgURE 8: FANOVA test for the regional disparity in the absolute level of FIE. (a) Functional mean. (b) Bootstrap resamples, $n=100$. (c) Density plot for bootstrap resamples; $N$ levels: 3 , stat $=1.949$, and $p$ value $=0.27$.

to be chosen; all these characteristics led to the fact that the ratio of credit demand in the eastern region is not as high as that in the western region [57]. Compared with the central region, the FIE level in the eastern region is higher than that in the central region from 2006 to 2015 , while the FIE level is basically lower than that in the central region after 2015.
From 2006 to 2015, the FIE level in the central region is the lowest among the three regions; however, due to the fact that the indirect financing efficiency in the eastern region in 2015 decreased, the FIE level in the central region is basically higher than that in the eastern region and lower than that in the western region after 2015. In 2012, Opinions on the 
Vigorous Promotion of the Rising Strategy for the Central Region by the State Council [63] was issued by the State Council; the preferential policy of the country is favorable for the central region to obtain more financial fund and transfer payment; besides, the Central Bank has approved more loans to the central region, which greatly increased the credit scale in the central region, so as to improve the FIE level in the central region. This stage corresponds to the trajectory of the FIE level in the central region from 2015 to 2018.

Looking into Figures 9 and 10, it is found that there is obvious difference among the speed and acceleration of the FIE level in the three regions; in this case, we may speculate that this is due to the difference in terms of the number of interregion financial intermediaries, the maturity of the market, and the human capital; in the meantime, the difference also exists in the development degree of the real economy, the opening degree of the policy environment, and perfection of the legal system, thus resulting in the big disparity in the growth potential of financing efficiency even for the united monetary system.

\subsection{Disparities' Decomposition}

3.2.1. Disparities' Decomposition of FSE. The regional financial disparities for the period 2006-2018 are illustrated in Figure 11. It can be seen from Figure 11(a) that the difference of FSE in the 11 provinces in the eastern region is relatively huge and mainly accounts for the formation of the overall disparity. The trajectory of the difference of FSE among the 11 provinces in the eastern region shows an obvious downtrend, indicating that the FSE difference among the provinces in the eastern region is greatly decreased; however, in the meantime, the above difference is far higher than that among the 10 provinces in the central region and that among the 9 provinces in the western region; therefore, the relatively large FSE difference among the 11 provinces in the eastern region mainly accounts for the formation of the overall FSE difference in China; however, the contribution rate provided by the 11 provinces in the eastern region is continuously decreasing. The decrease of the FSE difference in the eastern region is based on many reasons.

The provinces in the eastern region have the most developed real economy and wealth. With the further development of the economy, all provinces in the eastern region have a large demand for financial products. In addition, financial reform pilots are mostly spread out in the eastern provinces, providing financial vitality for the eastern provinces and enabling the financial industry to develop more evenly in the eastern region. Both points mentioned above lead to the convergence of the FSE difference in the eastern region and the further convergence of the FSE difference in China.

There are fluctuations among the 10 provinces in the central region; however, the change is not big in the long term, that is, a steadily rising status is maintained; the FSE difference among the 10 provinces in the central region was maintained at the steady upward trend from 2012 to 2014 and reached its extreme point in 2014 before it continued to decrease. In 2012, Opinions on the Vigorous Promotion of the Rising Strategy for the Central Region by the State Council was issued by the State Council; the period when the FSE difference among the 10 provinces in the central region was enlarged coincides with the period when this document was issued. The contribution to the national FSE overall difference from the 10 provinces in the central region is significantly lower than that from the 11 provinces in the eastern region; in the meantime, the contribution rate in terms of FSE difference of the 10 provinces in the central region is higher than that of the 9 provinces in the western region all the time except for the period from 2009 to 2013 when the contribution rate in the eastern region is slightly lower than that in the western region.

There are also fluctuations among the 9 provinces in the western region; however, the change is not big in the long term, that is, a steadily decreasing status is maintained; the FSE difference among the 9 provinces in the western region was maintained at the steady upward trend from 2007 to 2012; the first ten years in the 21st century are considered to be the period when the foundation for the implementation of the key national strategy of the Strategy for Large-Scale Development of Western China [64] of the country was laid; before the implementation of this national policy, the western region is like a desert for the economic development of China, and the financial development is in a chaos state. At this time, the financial development in the western region is still in its growth stage; therefore, the preferential policy needs time to be implemented; in this case, the provinces in the western region will receive the preferential policies with different degrees and at different times; therefore, the marginal benefits in terms of financial development scale for the provinces in the western region are rather high, and the sequential order of the time point to implement the policy also determined that the financial development scale within this period is extremely uneven. This period rightly coincides with the period when the FSE difference among the 10 provinces in the western region is steadily going up. Therefore, the FSE difference among the provinces in the central region and the western region is far below that in the eastern region, which indicates that the convergence of the FSE difference in China is mainly due to the fact that the difference in the eastern region is decreased.

3.2.2. Disparities' Decomposition of FSA. As for the trajectory of the FSA regional difference, there is no continuous increasing or declining trend, and the relative fluctuation amplitude among the three regions is not obvious.

It is worth noting that, as can be seen from Figure 11(b), the distribution of the FSA disparity among the 11 provinces in the eastern region has presented an inverted " $U$ " since 2007; the trajectory of the disparity in FSA for the eastern region shows an obvious downward trend since 2011, which indicates that the FSA disparity in the eastern region in China has decreased rapidly since 2011. In December 2006, the first foreign-owned security company in China-UBS Securities Co., Limited-was found in Beijing, which 


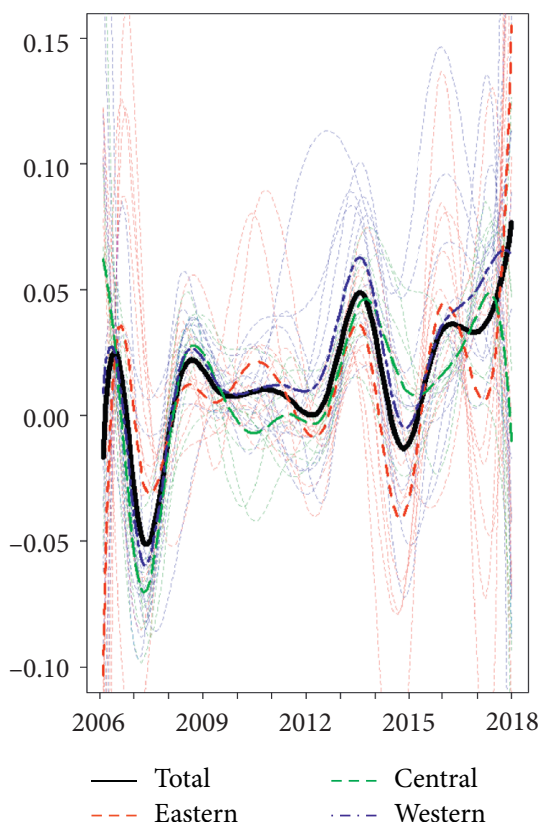

(a)

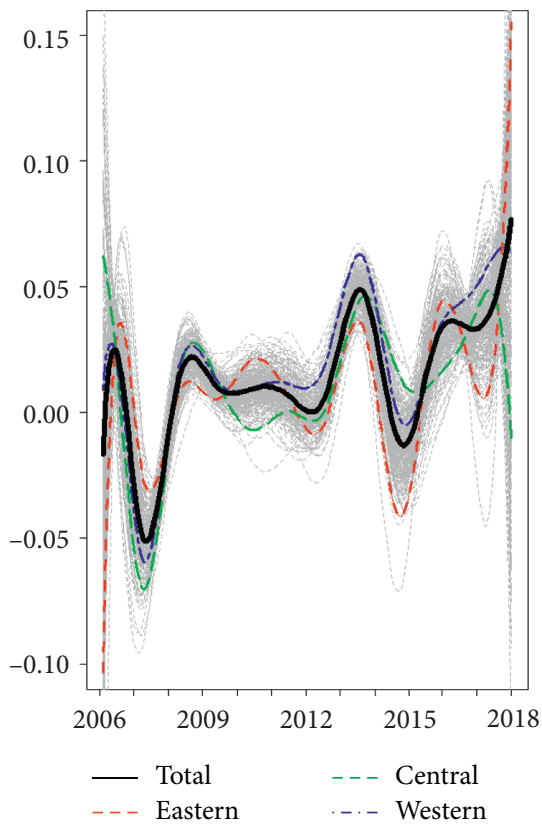

(b)

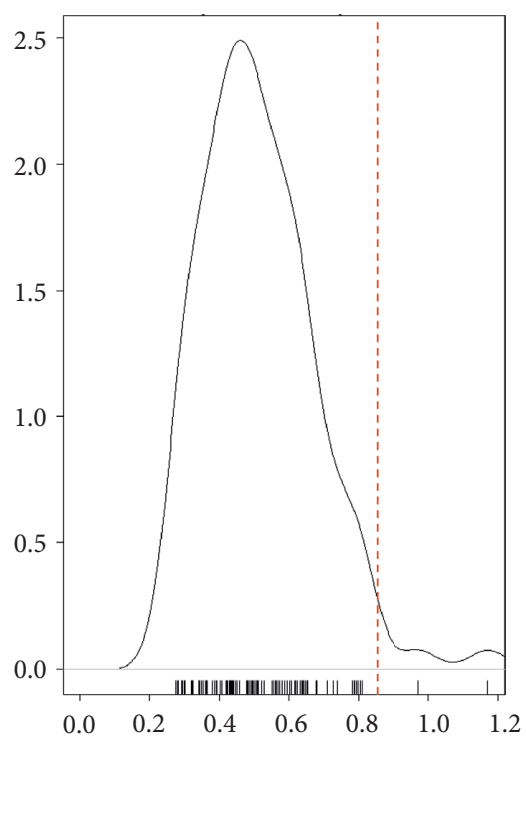

(c)

FIGURE 9: FANOVA test for the regional disparity in growth velocity of FIE. (a) Functional mean. (b) Bootstrap resamples, $n=100$. (c) Density plot for bootstrap resamples; $N$ levels: 3 , stat $=0.856$, and $p$ value $=0.02$.

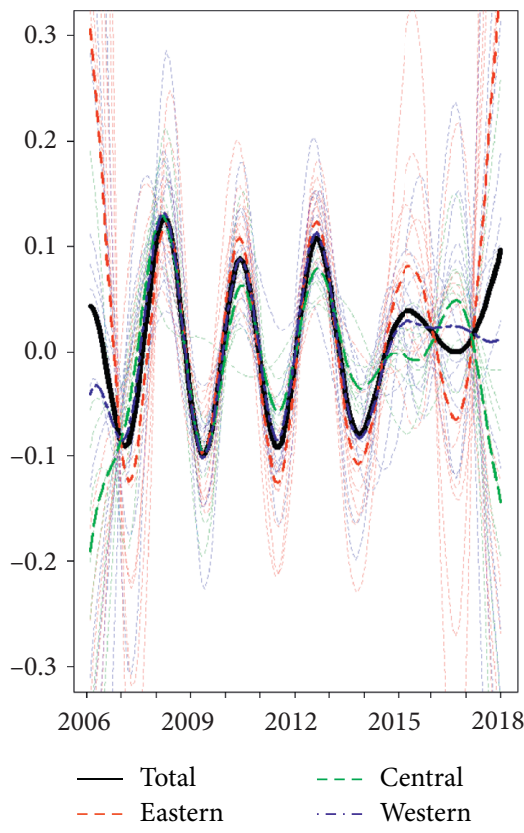

(a)

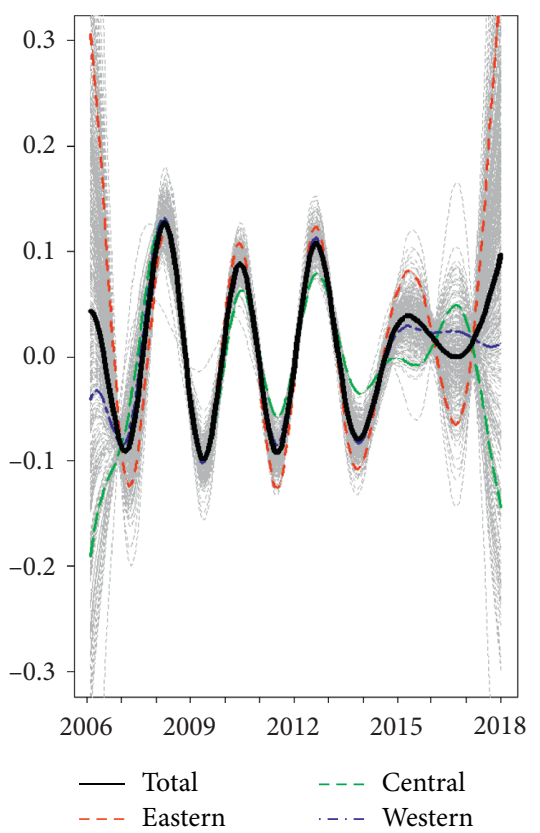

(b)

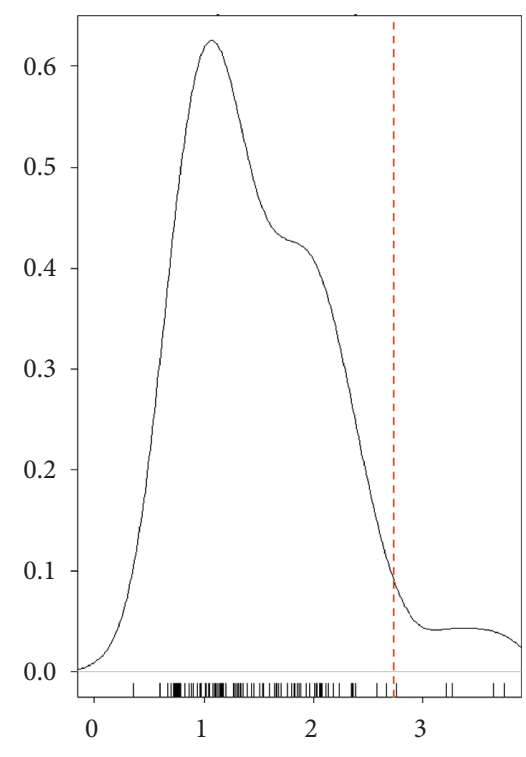

(c)

FIgURE 10: FANOVA test for the regional disparity in growth acceleration of FIE. (a) Functional mean. (b) Bootstrap resamples, $n=100$. (c) Density plot for bootstrap resamples; $N$ levels: 3 , stat $=2.723$, and $p$ value $=0.05$.

marked the new progress of financial opening-up in China. The foreign investment brings new vitality and management skills to the security industries; however, at the early stage of opening-up, foreign-owned security companies are only found in the most developed regions in the eastern region, such as Beijing; besides, at the early stage of the foundation of security companies, the radiation range only covers the city or province where it is located, rather than the surrounding regions; therefore, on the contrary, the FSA disparity in the eastern region has increased. The above process corresponds to the period from 2007 to 2011 when the FSA Theil index increased. Basically, the FSA Theil index 


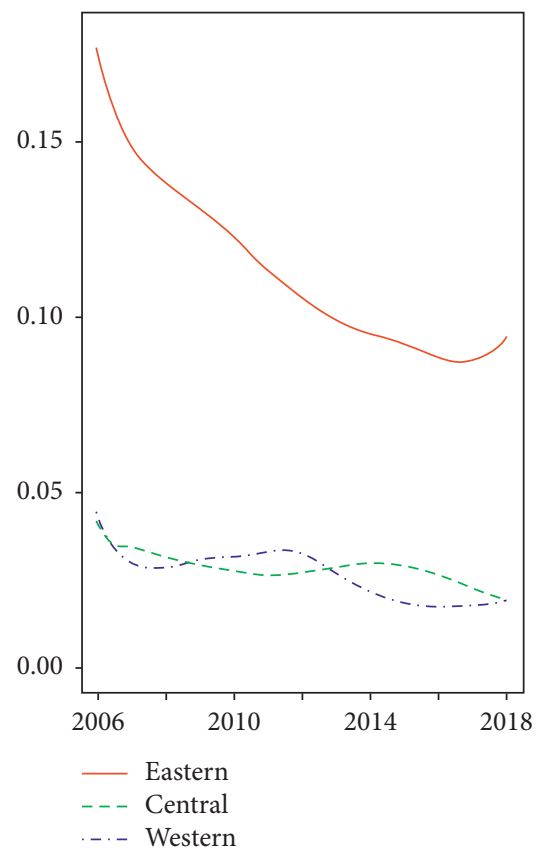

(a)

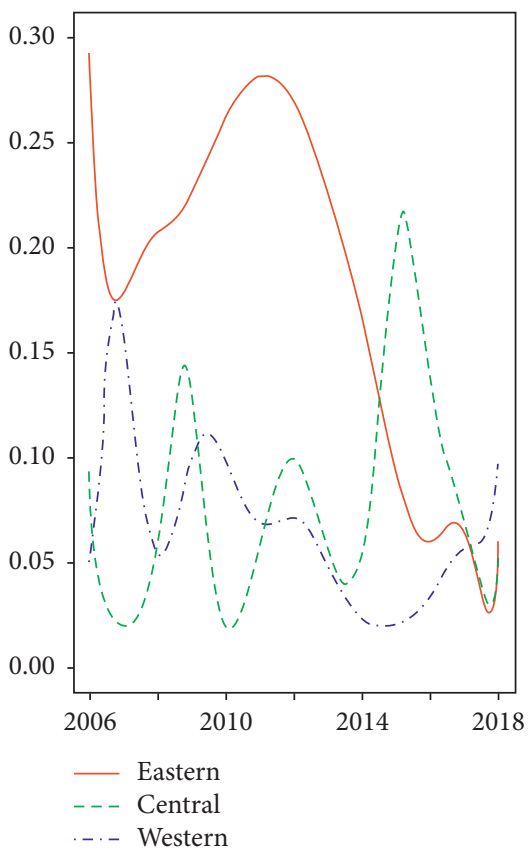

(b)

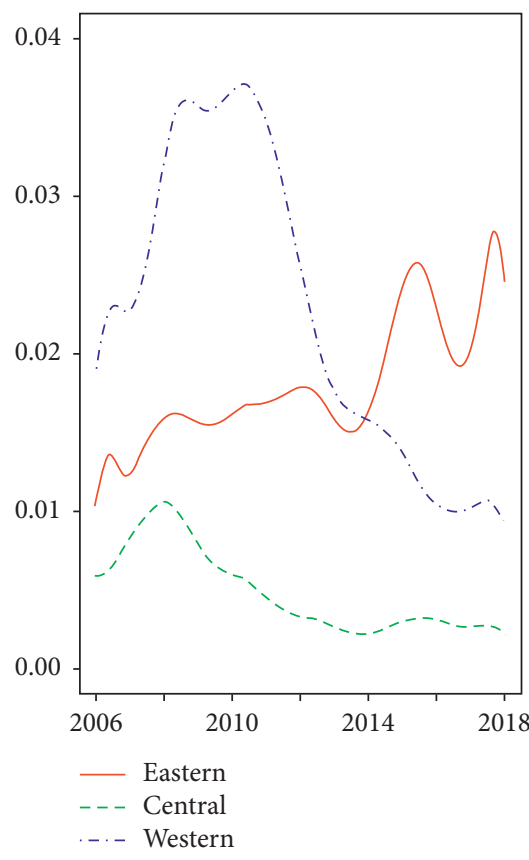

(c)

Figure 11: Functional Theil indexes for three dimensions of regional financial development. (a) Scale. (b) Structure. (c) Efficiency.

maintained a drastically declining trend from 2011 to 2018 with an oscillation in 2017 only, which indicates that the financial structure of the eastern region in China becomes more balanced after 2011. In 2011, the production and management condition as well as the financing of the micro-, small-, and medium-sized enterprises has drawn wide attention from all sectors of the society. The financial institutions take multifaceted measure to strengthen their financial service to the micro-, small-, and medium-sized enterprises, thus continuously enhancing the financial support to these enterprises. Opinions on Further Strengthening the Service to the Small and Medium-sized Enterprises [65] was printed and distributed by the People's Bank of China in June 2010. The eastern region is characterized by active economy and large quantity of private enterprises; the implementation of inclusive financial policies favorable for the development of the small- and medium-sized enterprises facilitates the development of the small- and medium-sized enterprises; in the meantime, the demand in the aspect of bond financing will increase as well, thus further lowering the FSA disparity among the 11 provinces in the eastern region. This corresponds to the steady decrease of the FSA disparity among the 11 provinces in the eastern region since 2011.

Different from the fact that the trajectory of the FSA Theil index among the 11 provinces in the eastern region presents the distribution of an approximate inverted " $U$," the Theil index of the 10 provinces in the central region and the 9 provinces in the western region presents a relatively drastic oscillation distribution on the time axis. The government factor and the market factor jointly decide the financial structure; because the adjustment by market factor is implemented step by step with certain inertia and time-lag effect in most cases, the impact brought by the market factor adjustment to FSA will not be violent [66]. However, the Theil distribution in the central region and the western region indicates that the financial structure in the central and western regions was subjected to the strong impact of the government factor.

3.2.3. Disparities' Decomposition of FIE. Compared with the above two dimensions, the regional difference of FIE shows different growth patterns. Specifically, the fluctuation of the FIE difference among the 11 provinces in the eastern region shows an upward trend. It can be seen from Figure 11(c) that the FIE difference among the 11 provinces in the eastern region rose dramatically around 2013. The year of 2013 is an important year for the financial reform of China when many pieces of big news were witnessed.

Release loan interest rate control further accelerates the marketization progress of interest rate [67]; Shanghai FreeTrade Zone was approved to be established, and financial reform has become the breakthrough point [68]. The above two major policies and measures were all seen in 2013, which started the further reform and opening of China.

All of them will lead to the expansion of the FIE difference among the 11 provinces in the eastern region, and this corresponds to the period around 2013 when the FIE difference rose dramatically among the 11 provinces in the eastern region.

The trajectory of the FIE difference among the provinces in the central and western regions decreased continuously at different speeds after it reached the peak value around 2010 . Besides, the trajectory of the FIE Theil index in the western region presents an overall inverted "U." The trajectory of the 
FIE level in the central and western regions is closely related to the fact that the country further pushes forward and deepens the strategy of Large-scale Development of China's Central and Western regions," so as to promote the balanced development of the financing efficiency among the provinces in the central and western regions [69].

Based on the functional Theil index, the data from the 31 provinces are used to calculate the total financial difference in three dimensions; besides, it is decomposed into inner-region difference and interregion difference. Though the trajectories of FSA and FIE suffer from the dramatic fluctuation, it can be seen from Figure 12 on the whole that all the total differences within the three regions present the declining trend. Specifically, all the three trajectories of the FSE difference present a similar declining trend regardless of the total difference, regional difference, or interregion difference, which indicates that the FSE level will come to a convergence. By contrast, the trend of the inner-region difference and interregion difference of FSA and FIE is completely different from the same fluctuation of FSE. The interregion difference of FSA is always maintained at a relatively low level, and the inner-region difference maintains a declining trend at a relatively high level; the overall difference is almost in parallel with the innerregion difference. The trend of the inner-region difference and interregion difference of FIE is also completely different from the same fluctuation of FSE. What is same as FSA is that the interregion difference of FIE is always maintained at a relatively low level, while what is different is that the innerregion difference maintains a trend in the shape of an approximate inverted " $U$ " at a relatively high level; the overall difference is almost in parallel with the inner-region difference.

However, a common characteristic that appeared in the total difference decomposition in three dimensions is that the inner-region difference is a main component of the total difference, while the share of the interregion is relatively small and stable. The relatively large inner-region difference indicates that the traditional zoning method in terms of the eastern region, the central region, and the western region among the provinces has been breached, that is, the financial development within the provinces in the same region is not homogenized, and the development on the financial level of different provinces within the same region is an extreme imbalance; this is an overturn of our awareness of the traditional zoning of the province. This phenomenon may be due to such a fact that the financial system and institutional arrangement of China are dominated by the government; in this case, the unified monetary and fiscal policy will lead to a very small gap between regions [70]. There is a big difference in the initial conditions for the financial development in 31 provinces; for example, even in the same region, there is still a big difference in terms of geographic position, development level of economy, number of financial intermediaries, expertise level of the financier, and maturity of the capital market [71]. However, with the gradual exiting of the government in the reform of the financial system, as well as the maturity of the market mechanism and the liberation of investment, the competition of the financial resources has changed from the state-owned resources to the market resources [72]. Therefore, all the differences in three dimensions showed the declining trend.

The policy implication concluded from the functional Theil index decomposition is that, with the advance of the reform and opening-up of China, the government financial control has been released gradually; due to the unrestricted flow of the financial resources, the Chinese market is becoming mature, and the government need not issue protective policies for the developing regions; instead, proper policy arrangement becomes necessary from the perspective of the provincial-level administrative region. When studying the regional financial difference in China, the research based on the provincial-level administrative region would be better to reflect the essence of the regional financial difference in China. The government has to inject vitality into the financial market, push forward the optimization and upgrading of the industrial structure in different regions, standardize the investment activities of the local government, further widen the financial channel, and construct the multilevel financial market [73].

\section{Summary}

The comparisons of the functional Theil index fluctuation shown in Figure 11 reveal that the disparities in the scale of financial development for three regions all exhibit a declining trend, whereas the drop of the eastern disparity far outweighs that of central and western disparities. In contrast, the trajectories of regional disparities in other two dimensions fluctuate violently, with no apparent rising or declining trend. Moreover, the magnitude of eastern disparities in the structure and efficiency of financial development is not always larger than that of the other two regions [74]. The above difference in disparities of financial development is consistent with the degrees of financial marketization in three regions. In fact, the development of the financial system is undoubtedly influenced by two forces, that is, the government and the market. Since the spatial sequence of opening-up and reform in China began from the coastal areas to the inland regions, the competition for financial resources in the eastern region has shifted from state-owned resources to market's ones. By contrast, financial resources in the central and western region mainly depend on stateowned resources; thus, the disparities of financial scale in the two regions have been obviously smaller. However, with financial liberalization being deepened, the regional disparities in the scale of financial development are narrowing. Due to the relatively low level of financial market development in central and western regions, bank credit is still the basic channel from saving into investment, whereas efficiency is not always the first goal of state-owned resource allocation in China; thus, the fluctuations of the financial structure and efficiency in central and western regions are more frequent [75].

The common rules inferred from disparities' decomposition of three dimensions shown in Figure 12 are that, no matter the total, intraregional, or interregional disparity, the FSE trajectories demonstrate a declining trend, indicating a countrywide convergence trend in the FSE level. In contrast, 


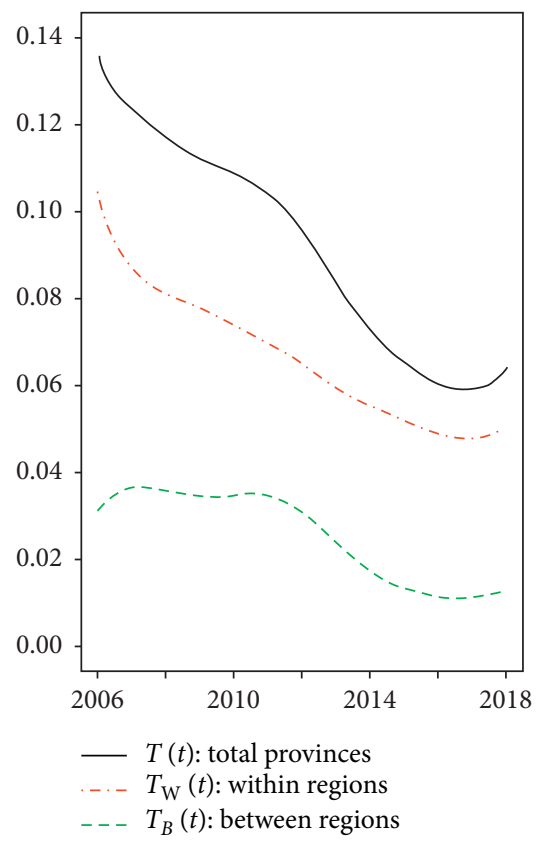

(a)

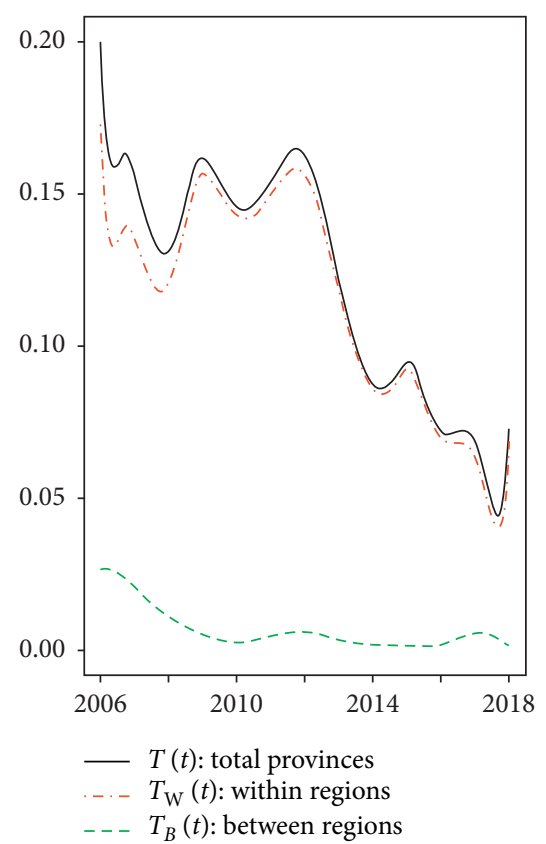

(b)

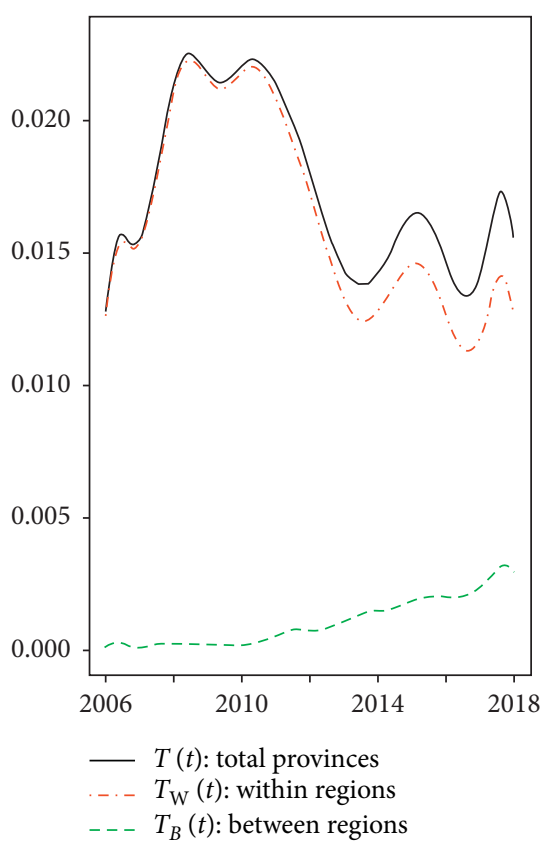

(c)

Figure 12: Disparities' decomposition for three dimensions of financial development. (a) Scale. (b) Structure. (c) Efficiency.

although the FSA trajectories also decline, sharp fluctuations are seen in the years of supply-side financial market reform. Interestingly, the trajectory of the interregional disparity of FIE reveals a slightly increasing trend, whereas total and intraregional disparities follow inverse U-shaped curves, with the turning point corresponding to the period of the "Plan of 4 Trillion Government Investment" after the 2008 financial crisis. Noticeably, a common feature is the dominance of intraregional disparities over interregional ones. Greater intraregional disparities indicate that provinces have broken the traditional regional division of eastern, central, and western. This can be attributed to the fact that since the financial system and institutional arrangements in China are mostly dominated by the government, uniform monetary and fiscal policies result in insignificant regional disparities. However, with gradual government withdrawal from the financial system, improvements in market mechanisms, and investments' liberalization, competition for financial resources has shifted from state-owned to market-owned resources, resulting in declining disparities for all three dimensions. Overall, the Chinese market is becoming mature, and financial resources are flowing freely; hence, the government does not need protective policies for underdeveloped regions.

\section{Conclusions and Policy Implications}

This paper reexamined three dimensions of financial disparities in China from static and dynamic perspectives using the FDA method. The findings are threefold. First, the increase in average levels and decline in the total variability among provinces for all three dimensions imply that financial reforms are reducing the regional disparity. Second, the differences in significance testing results reveal the persistence of regional differences in financial development, which may be attributed to the interaction between uniform financial systems dominated by the government and disparate regions' initial financial conditions. Third, the functional Theil index decomposition indicates that intraregional disparities dominate total disparity fluctuations. Since the overall financial disparities are declining while regional disparities still exist, financial liberalization should be further strengthened, although region-specific protective policies are no longer necessary. Further research should investigate the spatial-temporal convergence of the financial disparity using functional regression. Significant regional differences in financial development trajectories signify that a different modelling approach should be employed. The policy implications derived from the research studies are as follows.

5.1. Further Push Forward the Optimization and Upgrading of the Industrial Structure in Different Regions. To improve the social financing efficiency, it is necessary to further push forward the optimization and upgrading of the industrial structure in different regions [76]. As per the empirical results obtained from the central, western, and eastern regions, the ratio of the tertiary industry plays an important role in the improvement of social financing efficiency. On the contrary, the optimization and upgrading of the industrial structure is an important symbol for the realization of "economy servitization" (also called "tertiarization") under the promotion of the development of information technology and other factors; in other words, in the industrial scale, the proportion of service industry is greater than that of industry and becomes 
the focus of economic activity. In this case, it is necessary to carry out further innovation, perfect the development mechanism and system of the service industry, strive to develop modern service industries, including finance, insurance, logistics, information and legal service, accounting, intellectual property, technology, design, and consultation service, actively develop the industries with great potential, including culture, tourism, and community service, and accelerate the reform and development in the fields, including education and training, service for the aged, and health care. In the meantime, each region should invest the capital which is raised with the direct financing tools, such as loans and money management, trust, and leasing, saved by means of the incremental loans, vitalized existing loans, and asset securitization in the industries and programs in accordance with the national industrial policies and the policies for structural adjustment and upgrading. Meanwhile, pay attention to the structural optimization of different industries within the tertiary industry, and strengthen the support to the emerging tertiary industry; on the contrary, restrict the tertiary industry characterized by high ratio and rapid growth, such as real estate, and develop the key industries with appropriate priority, thus balancing and improving the investment structure of the tertiary industry, as well as the financing efficiency of the tertiary industry.

\subsection{Further Widen the Financing Channel and Construct a} Multilevel Capital Market. After the construction and development for many years, the Chinese multilevel capital market begins to take shape. However, on the whole, the development of our capital market still does not sound enough; the long-term existence of the problems of "financing difficulty and high cost" for the small- and medium-sized enterprises exposes the structural contradictions in our financing market; in this case, diversified financing platform should be further established. The first is to accelerate the development of the nonfinancing enterprise direct debt financing for the enterprise bond and interbank market; the second is to expand and develop the main board, middle, and small capital stock and the growth enterprise market while actively developing the new OTC market. While perfecting the new share issuance mechanism, refinancing mechanism, and delisting mechanism, as well as the merger and acquisition system, the governance mechanism of the listed companies should be improved, and the market transparency should be enhanced, thus strengthening the protection of the right and interest of the investors. The third is to perfect the insurance financing service system. In addition, different role definitions for different financing channels should be identified, thus realizing the differentiated cooperation between different markets and pushing forward the healthy and orderly development of the markets of the financing system [77]. At the same time, the government cannot ignore the role of the international capital market in promoting the development of China's financial market. However, as China continues to open up to the outside world and increase the use of foreign capital, it should also control the scale, proportion, and quality of foreign capital [78] in order to give full play to the inflow of foreign capital to China's financial development spillover effect.

\subsection{Further Standardize the Investment Activities of the Local} Government. As per the empirical study mentioned above, the investment activity of the local government to the central and western regions has increased the local credit scale, which actually generated certain crowding-out effects to the private investment. A large amount of social financing surges up into the investment programs obtained from the financing platform of the local government and the lowefficiency state-owned enterprises, which raised the social financing cost and led to the low efficiency of social financing. Therefore, the local government should standardize its investment activities and shift its focus from direct participation in the economy activities into the optimization of the market environment as well as the establishment and perfection of the market system gradually. Differentiate the public welfare program from the profit-oriented ones, strengthen budget assessment, and change the investment activities of the local government, that is, change the direct participation into indirect support. In the meantime, strengthen the supervision to the medium-to-large-scale state-owned enterprises, thus preventing the interest arbitrage activities by idle funds. Meanwhile, attention should be paid to the following problems: the first is to further perfect the market mechanism and push forward the marketization of the activities to obtain fund and capital elements by the state-owned enterprises; the second is to perfect the construction of the laws and regulations, contain the blind expansion and repeated construction by the state-owned enterprises, and standardize the market competition activities by the state-owned enterprises; the third is to perfect the internal control system in the state-owned enterprises and improve the investment decision-making procedure, thus preventing the blind investment characterized by low level and high risks.

\subsection{Further Detail the Regional Financial Policies in terms of} Preparation and Implementation. The current regional fiscal policy and development policy of the country are prepared and implemented based on the traditional zoning of the eastern, central, and western regions in most cases. However, through the research in this study, it is found that the innerregion difference of financial development is higher than the interregion difference; therefore, the traditional zoning of the eastern, central, and western regions is not targeting in terms of the preparation and implementation of the fiscal policy. The financial development of China should realize the transformation from the incremental development to quality improvement development. The old financial policies prepared and implemented based on the traditional zoning of large regions are no longer satisfactory for the requirement of high-quality financial development. The difference of the financial development in China comes from the innerregion difference; therefore, the government is required to breach the traditional and rough method for region zoning 
and adopt refined region zoning for the preparation and implementation within the allowable range of cost. The basis is to replace the big region with the provincial-level administrative unit which will be used as the basic main body for the country to plan, as a whole, the domestic financial development, as well as the preparation and implementation of the financial policies; replace the one-sidedness and incentive of the local government with the wholeness and connectivity of the overall planning for the financial development from the central government, thus realizing the higher-quality and balanced development of our finance.

\section{Data Availability}

The data used to support the findings of this study are included within the article.

\section{Conflicts of Interest}

The authors declare that they have no conflicts of interest.

\section{Acknowledgments}

This work was supported by the Natural Science Foundation of China (71701201 and 71603256) the Fundamental Research Funds for the Central Universities (no. 2021YCPY0112), and Jiangsu Key Laboratory of Coal-based Greenhouse Gas Control and Utilization (No. 2020KF02).

\section{References}

[1] S. S. Ba, "The development of capital market and the adjustment of financial structure-_also on the separation and integration of the banking industry and the securities industry," Contemporary Economic Sciences, vol. 20, no. 1, pp. 1-9, 1998.

[2] L. Zhou, Analysis of the Regional Disparity in China's Financial Development (1978-1999), South China Financial Research, Hong Kong, China, 2002.

[3] M. H. Zhan, "Comparative analysis of financial structure and economic development in different development stages," The Journal of Quantitative \& Technical Economics, vol. 20, no. 11, pp. 69-73, 2003.

[4] Z. P. Dong, "The characteristics, causes and countermeasures of China's unbalanced economic development," Social Science of Beijing, vol. 3, pp. 33-48, 2000.

[5] X. D. Lu, "Does the misallocation of financial resources hinder China's economic growth?" Journal of Financial Research, vol. 4, pp. 55-68, 2008.

[6] H. W. Zhou and Y. H. Zhong, "China's financial intermediary development and regional economic growth: a multivariate VAR system analysis," Journal of Financial Research, vol. 6, pp. 130-137, 2004.

[7] J. Gibson and R. Scott, Poverty and Access to Roads in Papua New Guinea, Economic Development and Cultural Change, Chicago, IL, USA, 2003.

[8] X. M. Wu, China Financial Development Theory, Economic Management Press, Beijing, China, 1994.

[9] R. Levine, Financial Development and Economic Growth: Views and Agenda, Social Science Electronic Publishing, Rochester, NY, USA, 1997.
[10] J. Zhang, "Regional differences in economy and financial growth," Finance and Economy, vol. 6, pp. 18-24, 1994.

[11] D. S. Yin and S. X. Xiao, Research on Regional Finance in System Transition, Xuelin Publishing House, Shanghai, China, 2000.

[12] F. C. Zhang and Q. R. Yuan, "A discussion on regional financial growth," Journal of Northeastern Normal University (Philosophy and Social Sciences Edition), vol. 1, pp. 58-63, 2001.

[13] S. G. Yang and H. Zhu, "The collapse of central China, the weakening of finance and the financial support for the rise of central China," Economic Research, vol. 42, no. 5, pp. 55-67, 2007.

[14] L. Zhou and Z. M. Wang, "An empirical analysis of financial development and economic growth in various regions of China: 1978-2000," Financial Research, vol. 10, pp. 1-13, 2002.

[15] A. G. Hu, "Challenge to unbalanced development-China: the political economy of unbalanced development," Leadership Decision Information, vol. 18, p. 32, 2000.

[16] J. Li, Research on the Differences in China's Regional Financial Development: Based on the Perspective of the Theory of Division of Labor, China Economic Press, Beijing, China, 2008.

[17] X. L. Dou and W. J. Wang, China's Regional Financial Development Differences, Changing Trends, and Local Government Behaviors-And on the Impact of Tax-Sharing Reforms on China's Regional Financial Differences, Shanghai Finance, Shanghai, China, 2011.

[18] Q. Q. Ye, Analysis on the Spatial Evolution of My Country's Regional Financial Development, Shanghai Finance, Shanghai, China, 2011.

[19] K. L. Zeng, "Must pay attention to the study of regional finance," Finance and Economics, vol. 4, pp. 15-18, 1995.

[20] J. Zhang, Economic Analysis of China's Financial Growth, China Economic Publishing House, Beijing, China, 1995.

[21] J. Z. Zhang, China Regional Financial Analysis, China Economic Publishing House, Beijing, China, 1995.

[22] W. X. Lu and G. P. Li, "Analysis of the convergence of China's regional financial development," Quantitative Economics and Technical Economics Research, vol. 2, pp. 125-128, 2004.

[23] X. J. Jin and L. Tian, "The trend of regional financial growth differences in my country: 1978-2003," Economic Theory and Economic Management, vol. 8, pp. 24-30, 2004.

[24] W. Zhao and R. Y. Ma, “The differences in China's regional financial growth-_based on the measurement of theil index," Economic Geography, vol. 1, pp. 11-15, 2006.

[25] M. Fujita and D. Hu, "Regional disparity in China 1985-1994: the effects of globalization and economic liberalization," The Annals of Regional Science, vol. 35, no. 1, pp. 3-37, 2001.

[26] Z. Chen and K. E. Haynes, "Impact of high-speed rail on regional economic disparity in China," Journal of Transport Geography, vol. 65, pp. 80-91, 2017.

[27] L. L. Qu, "China's multi-level financial center system construction," State-owned Assets Management, vol. 12, pp. 49-50, 2009.

[28] R. S. Tsay, "Some methods for analyzing big dependent data," Journal of Business and Economic Statistics, vol. 34, no. 4, pp. 1-47, 2016.

[29] R. W. Goldsmith, Financial Structure and Development, Yale University Press, New Haven, CT, USA, 1969.

[30] C. Y. Sun and J. H. Luo, "Analysis of the impact of regional resource allocation efficiency and financial efficiency on regional differences," Journal of Chengdu Administration Institute, vol. 3, pp. 45-49, 2014. 
[31] T. Beck, "Financial development and international trade," Journal of International Economics, vol. 57, no. 1, pp. 107-131, 2002.

[32] W. P. Osterberg, “Tobin's Q, investment, and the endogenous adjustment of financial structure," Journal of Public Economics, vol. 40, no. 3, pp. 293-318, 1989.

[33] P. L. Brockett, "Financial intermediary versus production approach to efficiency of marketing distribution systems and organizational structure of insurance companies," Journal of Risk and Insurance, vol. 72, no. 3, pp. 393-412, 2005.

[34] S. Wang, M. Jia, and Y. Zhou, "Impacts of changing urban form on ecological efficiency in China: a comparison between urban agglomerations and administrative areas," Journal of Environmental Planning and Management, vol. 63, no. 10, pp. 1834-1856, 2020.

[35] J. O. Fan and J. B. Ramsey, "Functional data analysis of the dynamics of the monthly index of nondurable goods production," Journal of Econometrics, vol. 107, no. 1, pp. 327-344, 2002.

[36] J. O. Ramsay and B. W. Silvermann, "Functional data analysis. springer series in statistics," Biometrical Journal, vol. 40, p. 56, 2010.

[37] J. O. Ramsay, "Confidence regions for multidimensional scaling analysis," Psychometrika, vol. 43, pp. 145-160, 1978.

[38] J. O. Ramsay and B. W. Silvermann, "Functional data analysis: springer series in statistics (p56)," Biometrical Journal, vol. 40, no. 1, pp. 56-56, 2020.

[39] S. S. Shaphiro and M. B. Wilk, "An analysis of variance test for normality (complete samples)," Biometrika, vol. 52, pp. 591611, 1965.

[40] H. Theil, Economics and Information Theory. Success in Economics, ScienceDirect, Amsterdam, The Netherlands, 1967.

[41] C. L. Cheng and G. H. Hua, "The dynamic changes of Jiangsu's financial development gap: 2001-2011-based on the analysis of Theil inde, x" East China," Economic Management, vol. 27, no. 11, pp. 22-25, 2013.

[42] X. Z. Chen, "An analysis of regional financial development differences in Guangdong province based on theil index method," Chinese Market, vol. 2, pp. 6-9, 2020.

[43] Y. H. Zhu, "The structure and efficiency of financial resource allocation in China-based on theil index," Technical Economics and Management Research, vol. 6, no. 325, pp. 62-67, 2017.

[44] Y. Z. Zheng, "Evaluation and analysis of financial agglomeration level in Yangtze River Delta and Beijing-Tianjin-Hebei economic zone in China-_comprehensive evaluation method of topsis index and decomposition of theil index based on grey relation," Journal of Fuqing Branch of Fujian Normal University, vol. 1, pp. 32-52, 2020.

[45] C. D. Zheng, "On the regional difference in financial development in China: based on the method of theil indexes," The Theory and Practice of Finance and Economics, vol. 29, no. 4, pp. 7-13, 2008.

[46] H. Liang, "Analysis on the regional differences of China's financial development and its causes-based on the decomposition of theil index," Modern Business Trade Industry, vol. 36, no. 11, pp. 1-4, 2015.

[47] C. Hsiao, "Evaluating the effectiveness of China's financial reform-The efficiency of China's domestic banks," China Economic Review, vol. 35, no. 4, pp. 70-82, 2015.

[48] S. F. Bian, "Research on the evaluation of the industrial development level of the three eastern economic regions,"
Doctoral Dissertation, Hefei University of Technology, Hefei, China, 2013.

[49] F. Cai and Y. Du, "Regional disparity, convergence and western development," China Industrial Economics, vol. 2, pp. 48-54, 2001.

[50] Z. J. Hu and C. J. Feng, "Foreign policies to promote scientific and technological progress and innovation," Science \& Technology Progress and Policy, vol. 1, pp. 22-28, 2006.

[51] Y. S. Chen, "Political relations, institutional environment and capital investment of listed companies," Journal of Finance and Economics, vol. 35, no. 12, pp. 27-39, 2009.

[52] F. Allen, J. Qian, and M. Qian, "Law, finance, and economic growth in China," Journal of Financial Economics, vol. 77, no. 1, pp. 57-116, 2005.

[53] Y. H. Bu, X. M. Zhao, and F. Yang, "Research on the model, impact and supervision of high leverage in China's commercial bank interbank business," Financial Regulation Research, vol. 2, pp. 33-46, 2014.

[54] P. G. Zhang and T. Chan, China's Capital Market. The Chinese Yuan: Internationalization and Financial Products in China, Edward Elgar, Cheltenham, UK, 2012.

[55] K. Yang, "Research on the spatial distribution of Chinese listed companies," Contemporary Economics, vol. 35, no. 467, pp. 44-45, 2017.

[56] X. Liu, "Comparative research on the policies of promoting the development of financial industry in various provinces and cities of China," Finance \& Economy, vol. 6, pp. 137-139, 2014.

[57] Y. M. Yan, "The path and model of financial service industry cluster: a comparative study of the three economic circles in eastern China. reform and innovation: China's development and China's future," in Proceedings of the The 10th Annual Conference of Shanghai Social Sciences, Shanghai, China, October 2015.

[58] H. F. Mao, "Prescribe the right medicine" for the industrial transformation in the Midwest," Financial World, vol. 2, pp. 108-109, 2016.

[59] Z. L. Li, "State-owned enterprise debt management and bank credit risk," Finance \& Economics, vol. 1, pp. 99-101, 1999.

[60] S. Y. Ma and H. X. Yu, "Transfer payment and regional economic convergence," Economic Research Journal, vol. 3, pp. 26-33, 2003.

[61] H. K. Wei, "Adjustment and prospect of China's national regional policy," Theoretical Reference Development Policy, vol. 17, no. 5, 2009.

[62] L. G. Wang and W. S. Zhao, "Research on my country's financial development and industrial structure upgrade," Research on Financial and Economic Issues, vol. 1, pp. 22-29, 2015.

[63] "Opinions on the vigorous promotion of the rising strategy for the central region," Bulletin of the State Council of the People's Republic of China, vol. 25, pp. 21-29, 2012.

[64] W. D. Liu, "China regional development report: the trend of western development," 2009.

[65] "Opinions on further strengthening the service to the small and medium-sized enterprises," Chinese SMEs9, vol. 9, 2010.

[66] B. Podobnik, "Time-lag cross-correlations in collective phenomena," EPL (Europhysics Letters), vol. 90, no. 6, pp. 1632-1652, 2010.

[67] H. J. Li, "The challenge of the marketization of interest rate to commercial banks and the countermeasures," Studies of International Finance, vol. 339, no. 2, pp. 65-76, 2015.

[68] J. Wu, "China (Shanghai) free trade zone financial reform outlook," New Finance, vol. 11, pp. 12-15, 2013. 
[69] F. Li, "An empirical analysis of financial disintermediation, financing structure differences and economic growth-_based on the empirical evidence of eastern, central and western regional economy," Hainan Finance, vol. 10, pp. 8-12, 2012.

[70] G. A. Zeng and Y. J. Ma, "The influences of financial structure in economic growth of the east, middle and west regions: empirical analysis based on China's province panel data," Economic Issues, vol. 9, pp. 40-46, 2017.

[71] S. S. Mo, "Financial restructuring and economic development from the perspective of Institutional arrangements in China," Doctoral Dissertation, Zhejiang University, Hangzhou, China, 2018.

[72] F. B. Sun, "China's regional financing structure difference impact on regional economic growth," Doctoral Dissertation, Peking University, Beijing, China, 2017.

[73] Y. F. Zhang and Y. Q. He, "Empirical analysis of listed companys'financing efficiency difference in central region," Journal of Nanchang University (Science Edition), vol. 37, no. 4, pp. 334-338, 2013.

[74] X. J. Zhang, "Research on the difference of financing structure and financing efficiency among regions in China," Journal of Regional Financial Research, vol. 12, pp. 77-84, 2015.

[75] X. X. He and L. Zhang, "The disparities of financial structures among different regions in China," Financial Forum, vol. 15, no. 1, pp. 25-31, 2010.

[76] P. He, D. D. Chen, and X. Y. Jian, "Research on optimization of industrial structure," Statistical Research, vol. 31, no. 7, pp. 31-37, 2014.

[77] H. Robert, Capital-Market Imperfections and Investment, Social Science Electronic Publishing, Rochester, NY, USA, 1998.

[78] F. Fan, H. Lian, X. Y. Liu, and X. L. Wang, "Can environmental regulation promote urban green innovation efficiency? an empirical study based on Chinese cities," Journal of Cleaner Production, vol. 287, Article ID 125060, 2021. 